\title{
High-resolution terahertz spectroscopy with quantum-cascade lasers
}

Cite as: J. Appl. Phys. 125, 151401 (2019); https://doi.org/10.1063/1.5084105

Submitted: 04 December 2018 . Accepted: 14 March 2019. Published Online: 08 April 2019

H.-W. Hübers (D), H. Richter (D), and M. Wienold (D)

\section{COLLECTIONS}

Paper published as part of the special topic on Advances in Terahertz Solid-State Physics and Devices
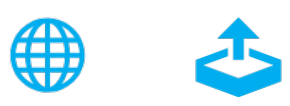

\section{ARTICLES YOU MAY BE INTERESTED IN}

On-chip mid-infrared and THz frequency combs for spectroscopy

Applied Physics Letters 114, 150401 (2019); https://doi.org/10.1063/1.5097933

Terahertz quantum-cascade lasers for high-resolution spectroscopy of sharp absorption lines Journal of Applied Physics 125, 151613 (2019); https://doi.org/10.1063/1.5079701

Potential clinical applications of terahertz radiation

Journal of Applied Physics 125, 190901 (2019); https://doi.org/10.1063/1.5080205

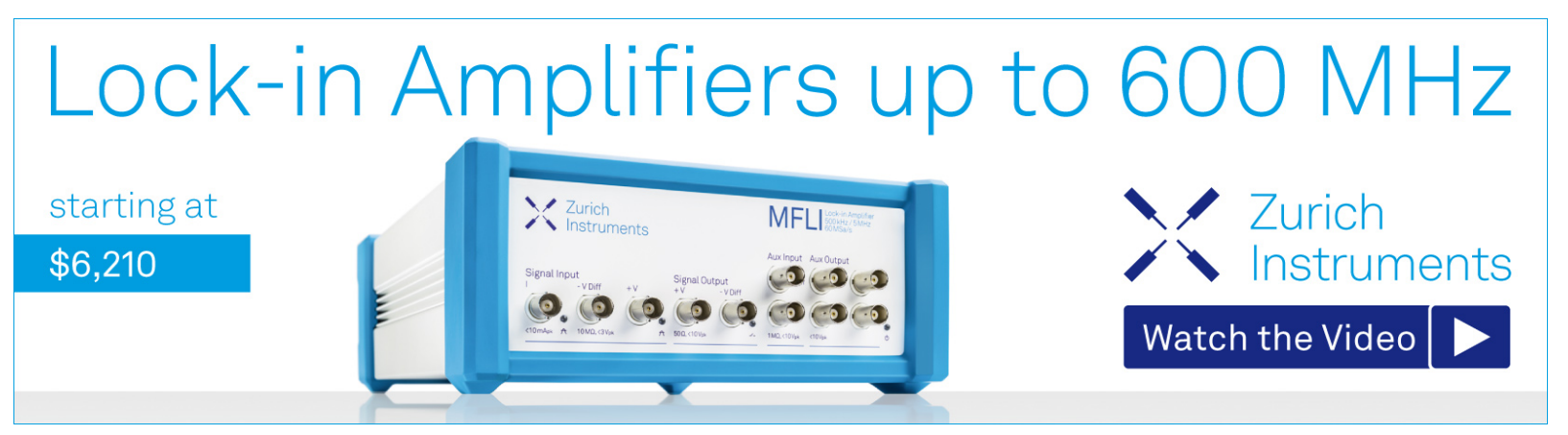




\title{
High-resolution terahertz spectroscopy with quantum-cascade lasers
}

\author{
Cite as: J. Appl. Phys. 125, 151401 (2019); doi: 10.1063/1.5084105 \\ Submitted: 4 December 2018 . Accepted: 14 March 2019. \\ Published Online: 8 April 2019
}

H.-W. Hübers, ${ }^{1,2, a)}$ (D) H. Richter, ${ }^{1}$ (D) and M. Wienold ${ }^{1,2}$ (D)

\begin{abstract}
AFFILIATIONS
${ }^{1}$ Institute of Optical Sensor Systems, German Aerospace Center (DLR), Rutherfordstraße 2, 12489 Berlin, Germany

${ }^{2}$ Department of Physics, Humboldt-Universität zu Berlin, Newtonstraße 5, 12489 Berlin, Germany
\end{abstract}

a)Author to whom correspondence should be addressed: heinz-wilhelm.huebers@dlr.de

\begin{abstract}
Terahertz (THz) quantum-cascade lasers (QCLs) are narrow band, high-power, and frequency-agile sources. These properties make them attractive for applications in high-resolution molecular and atomic spectroscopy. In the past few years, various techniques and methods regarding high-resolution spectroscopy with THz QCLs have been developed, namely, direct absorption spectroscopy, detection schemes such as wavelength and frequency modulation, differential spectroscopy, photoacoustic spectroscopy, and heterodyne spectroscopy. We briefly review the most relevant technological and methodological advances in this field, and we discuss perspectives and future directions.
\end{abstract}

Published under license by AIP Publishing. https://doi.org/10.1063/1.5084105

\section{INTRODUCTION}

Terahertz $(\mathrm{THz})$ molecular spectroscopy is fundamental for unveiling the structure and energy levels of molecules and atoms. Information on Doppler and pressure broadening can be obtained from their $\mathrm{THz}$ spectra. ${ }^{1}$ These data are a prerequisite for the interpretation of molecular spectra obtained in atmospheric science and astronomy. ${ }^{2}$ Molecular absorption lines are produced by transitions between rotational states of the molecule. Typically, light-weight molecules such as $\mathrm{CO}$ or $\mathrm{OH}$ have most of their rotational transitions at $\mathrm{THz}$ frequencies. In the case of atoms, transitions occur within a multiplet of orbital angular momentum, whose states are split by the spin-orbit interaction. These are called fine structure lines. A prominent example is the OI fine structure line at $4.7448 \mathrm{THz}$, which is important in astronomy and atmospheric science.

$\mathrm{THz}$ quantum-cascade lasers (QCLs) are important for highresolution molecular spectroscopy (for simplicity, we will use the term "molecular" instead of "molecular and atomic"), because they are powerful, narrow band sources and their spectral brightness is very high, typically in the order of $\mathrm{mW}$ per MHz. THz QCLs have been implemented in absorption spectrometers as well as in heterodyne spectrometers. In absorption spectroscopy, the goal is to detect a small fractional change of a relatively large amount of power. Heterodyne spectrometers are mostly used for remote sensing in astronomy and in atmospheric science. In this case, a very weak signal on a low background has to be detected. In a heterodyne spectrometer, the QCL serves as a local oscillator (LO) for the mixer, which downconverts the signal from the $\mathrm{THz}$ range to a few $\mathrm{GHz} .^{3}$ Although both applications are different, the requirements regarding the QCL are similar. With respect to its frequency characteristics, the QCL should provide large frequency coverage while having a narrow linewidth. Its power should be large enough to pump the mixer, while the power stability should be sufficient in order not to introduce additional noise in the spectrometer. The beam emitted by the QCL should be a fundamental Gaussian, because this relaxes the requirements regarding the optics of the spectrometer, independently whether it is an absorption spectrometer or a heterodyne spectrometer.

Until now, there are many reports about absorption spectrometers with $\mathrm{THz}$ QCLs. These are mainly focused on the development of spectroscopic methods, while routine applications are rare. However, many spectroscopic methods that are necessary for highresolution molecular spectroscopy have been demonstrated with $\mathrm{THz}$ QCLs. Examples are frequency and wavelength modulation, ${ }^{4}$ differential spectroscopy, ${ }^{5}$ photoacoustic spectroscopy, ${ }^{6}$ frequencycomb assisted spectroscopy, ${ }^{7}$ and saturation spectroscopy. ${ }^{8}$ It appears that the time has come for implementing $\mathrm{THz}$ QCLs in spectrometers for routine measurements.

In 2014, the first THz QCL LO became operational. ${ }^{9}$ It is part of GREAT, the German Receiver for Astronomy at Terahertz Frequencies ${ }^{10}$ on board of SOFIA, the Stratospheric Observatory for 
Infrared Astronomy. The LO has been developed for observations of the OI emission at 4.7 THz. In 2016, an upgrade of the LO has been implemented, which is capable of pumping seven hot-electron bolometric mixers instead of only one as for the first generation LO. ${ }^{11}$ These LOs are now routinely used on board of SOFIA. Besides for SOFIA, QCL LOs are under consideration for a number of balloon or spaceborne missions. These are GUSTO, the Galactic/Extragalactic Ultra long Duration Balloon Spectroscopic-Stratospheric Terahertz Observatory; ${ }^{12}$ LOCUS, the Low-Cost Upper-Atmosphere Sounder; ${ }^{13}$ FIRSPEX, the Far Infrared Spectroscopic Explorer for probing the lifecycle of the interstellar medium; ${ }^{14}$ and HERO, the Heterodyne Receiver for the Origins Space Telescope. ${ }^{15,16}$ With these highlevel missions at the horizon, the development of THz QCLs for applications as LOs becomes even more pressing. This includes not only the laser performance but also technical issues such as space qualification.

In this Perspective article, we focus on the application of $\mathrm{THz}$ QCLs in high-resolution spectroscopy for both absorption spectrometers and heterodyne spectrometers. We will briefly review the state-of-the-art regarding QCLs and their applications in spectroscopy and provide an outlook on future developments.

\section{HIGH-RESOLUTION SPECTROSCOPY: INSTRUMENT CONSIDERATIONS}

A simplified scheme of an absorption spectrometer is shown in Fig. 1. The emission of the QCL is guided by some optical elements through an absorption cell and the transmitted radiation is focused on a detector. In order to obtain a spectrum of the molecules in the absorption cell, the frequency of the QCL is swept across a certain frequency range, for example, by changing the driving current and/ or the temperature of the QCL. The sensitivity of the spectrometer can be increased by frequency or wavelength modulation of the QCL. In this case, the If or $2 \mathrm{f}$ signal is detected with a lock-in amplifier. The goal of absorption spectroscopy is to detect a small fractional change of power, $\Delta \mathrm{P}$, induced, for example, by a weak molecular transition, on the background of a large amount of

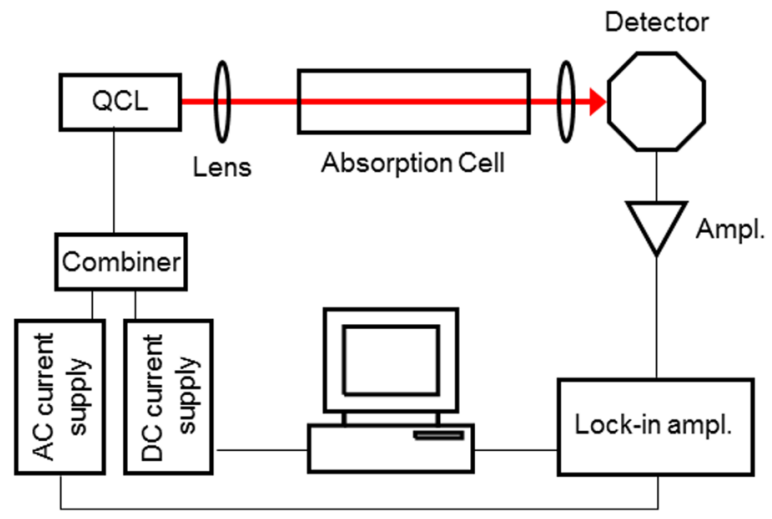

FIG. 1. Scheme of an absorption spectrometer with QCL. Wavelength and/or frequency modulation is enabled by the $\mathrm{AC}$ current source and the lock-in amplifier. source power, $\mathrm{P}_{\mathrm{S}}$. Ideally, the minimum detectable absorption, $\Delta \mathrm{P} /$ $\mathrm{P}_{\mathrm{S}}$, is limited for a given source power by the noise of the detector. Obviously, high source power is advantageous, although the improvement is less dramatic as it seems at first glance, since many detectors are photon noise limited, i.e., the noise-equivalent power (NEP) is proportional to $\sqrt{ } \mathrm{P}_{\mathrm{S}}$. For a source with $1 \mathrm{~mW}$, the minimum detectable absorption can be as low as $10^{-9} .{ }^{17}$ In practice, this is difficult to achieve since standing waves and source power fluctuations limit the sensitivity of the absorption spectrometer.

In a heterodyne spectrometer, a weak $\mathrm{THz}$ signal, for example, from an interstellar molecular cloud is detected by downconverting it to a few $\mathrm{GHz}$ (Fig. 2). This is done in a nonlinear detector, the so-called mixer, which produces the difference frequency between the LO and the signal radiation. Both are spatially superimposed, for example, by a beam splitter, which transmits/reflects most of the signal radiation to the mixer, while most of the $\mathrm{LO}$ radiation is lost. If LO power is scarce and this loss is not acceptable, a diplexer is used at the expense of somewhat higher optical loss for the precious signal radiation. For a QCL as for any other LO, the power requirement is determined by the type of mixer, the number of mixers, and the optical coupling of LO and signal radiation to the mixer. The domain of QCLs as LO sources is above about $3 \mathrm{THz}$, where powerful multiplier-based solid-state sources are not available. For example, an array with 14 liquid-helium cooled hot-electron bolometer (HEB) mixers and beam-splitter coupling requires about $1-2 \mathrm{~mW}$ from the LO source (assuming $1 \mu \mathrm{W}$ for pumping the HEB mixer and $99 \%$ loss of LO radiation at the beam splitter). If cooling with liquid helium is not an option, Schottky diode mixers are the best choice. However, such a mixer needs approximately $1 \mathrm{~mW}$ of LO power. If several Schottky diodes have to be pumped by the LO or if beam-splitter coupling is envisaged, the LO power requirements increases approximately by a factor of ten, which is challenging for QCLs but feasible.

Another important parameter of a spectroscopic source is its emission linewidth. It has to be significantly smaller than the transition linewidth, which is to be measured. The width of molecular rotational transitions is determined by the velocity or translational kinetic energy of the molecule and by the pressure. The Doppler width of their transitions is typically in the order of a few MHz. Broader lines are observed when the pressure in the absorption cell leads to a collision-induced broadening. This broadening depends strongly on the molecular species. Typical values are a few $\mathrm{MHz}$ per hPa. Cooling of the molecules will lower their translational energy and reduce the Doppler linewidth, but due to the $\sqrt{T}$ dependence (T: temperature) of the Doppler linewidth, the narrowing is rather limited. Lamb-dip (saturation) spectroscopy allows for a spectral resolution well below the Doppler limit down to about $50 \mathrm{kHz} .{ }^{18}$ Observation of a Lamb-dip requires the saturation of the transition, which in turn requires a high-power source. This makes high-precision Lamb-dip spectroscopy especially challenging at frequencies well above $1 \mathrm{THz}$ where powerful sources are lacking. For example, using a multiplier source with about $10 \mu \mathrm{W}$ output power at $2.6 \mathrm{THz}$ has enabled the observation of a Lamb-dip of a $\mathrm{H}_{2} \mathrm{O}$ transition. However, it was observed with a signal-to-noise ratio (SNR) of four and the measurement time was $17 \mathrm{~h} .{ }^{19}$ When the molecule's motion along the spectroscopic beam is much smaller than the wavelength, the rotational Lamb-Dicke regime is reached. 


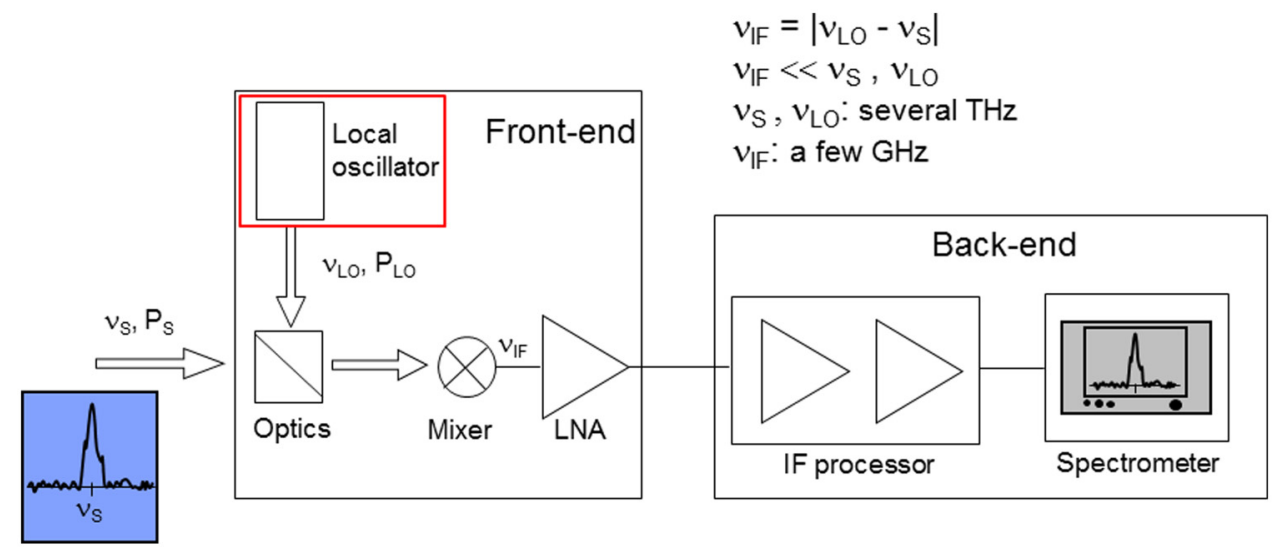

FIG. 2. Scheme of a heterodyne spectrometer. The QCL is used as a local oscillator ( $v_{I F}$ intermediate frequency; $v_{\mathrm{LO}}$, LO frequency; $v_{\mathrm{S}}$, signal frequency; LNA, low-noise amplifier).

Recently, this has been realized for trapped $\mathrm{HD}^{+}$obtaining a linewidth as low as $1.3 \mathrm{kHz}$ at $1.3 \mathrm{THz}$ using a multiplier source. ${ }^{20}$ With respect to linewidth, the requirements for a LO in a heterodyne spectrometer are very similar to those of an absorption spectrometer, because the spectral properties of the LO are imprinted one-to-one onto the downconverted spectrum.

Regarding the frequency coverage of a spectroscopic source, typically ten times the width of the absorption line is sufficient. However, in laboratory spectroscopy, a large spectral coverage is desirable in order to measure many transitions of the molecule under investigation without changing the source. It should be noted that the precision of the determination of the structural parameters of a molecule improves with increasing number of measured absorption lines. From a practical point of view, a fractional bandwidth of $10 \%-20 \%$ of the center frequency is quite adequate, because this enables one to cover the whole $\mathrm{THz}$ spectral region with a few sources. In the case of a heterodyne spectrometer, the instantaneous frequency coverage is given by the bandwidth of the mixer or the intermediate frequency (IF) processor. This is typically a few GHz. However, when different molecules have to be measured, the frequency of the LO has to be tuned into the vicinity of the molecular frequency. As for absorption spectrometers, a frequency coverage of $10 \%-20 \%$ is therefore desirable in order to cover many lines with one LO. In any case, the frequency tunability should be at least a few $\mathrm{GHz}$, because this covers the Doppler shift of most astronomical objects in our galaxy.

Another important parameter for any spectroscopic system is the beam profile of the source. In general, the optics of a spectrometer is designed for the fundamental Gaussian mode, because this mode has the smallest transverse dimension. Therefore, it allows using small optical elements and it reduces the loss of source power due to diffraction. For a heterodyne spectrometer, another issue is even more important. The mixer is an antenna-coupled device and the antenna is designed to accept a fundamental Gaussian mode. Any mismatch between the modes of the mixer antenna and the beam launched by the source, for example, by a nonGaussian beam profile of the source, leads to a reduction of the useful pump power of the LO. Moreover, the LO power, which is not in the fundamental mode, might be reflected by the mixer and generates standing waves and optical feedback, which affects the power and frequency stability of the QCL. ${ }^{21}$ Since heterodyne spectrometers are often used outside the laboratory under changing environmental conditions, the standing waves may also change on rather short time scales. Therefore, a beam profile as close as possible to a fundamental Gaussian mode is even more important in a heterodyne spectrometer than in a laboratory absorption spectrometer.

\section{THz QUANTUM-CASCADE LASERS}

\section{A. Some fundamentals}

The fundamentals of $\mathrm{THz}$ QCLs are covered by several reviews, ${ }^{22-24}$ and a comprehensive overview of the involved physics is given in the textbook of Faist. ${ }^{25}$ Here, we will briefly summarize the most important aspects with respect to high-resolution spectroscopy. As any other laser, THz QCLs are made of a gain medium and a resonating cavity. Optical gain is provided by a complex quantum-well structure, the active region. Population inversion is obtained between confined electronic subband states when an electrical bias is applied. The active-region structure is typically $10-15 \mu \mathrm{m}$ thick, containing several thousands of layers with thicknesses in the nanometer and sub nanometer range. The demanding epitaxial growth is usually realized by molecular-beam epitaxy and can take up to one day. ${ }^{26}$ There exist several strategies for the design of the active region. Inherent to virtually all QCLs is the use of a periodic sequence in which electrons undergo a cascade of many radiative transitions. This fundamental concept exploits the fact that QCLs are unipolar devices for which electron-hole recombination is absent. Note that even a perfectly periodic quantum-well structure does not imply that each period behaves the same. Charge accumulation due to the presence of negative differential resistance can result in a splitting of the active region into high- and low-electric field domains. ${ }^{27-29}$ Due to the nature of intersubband transport, 

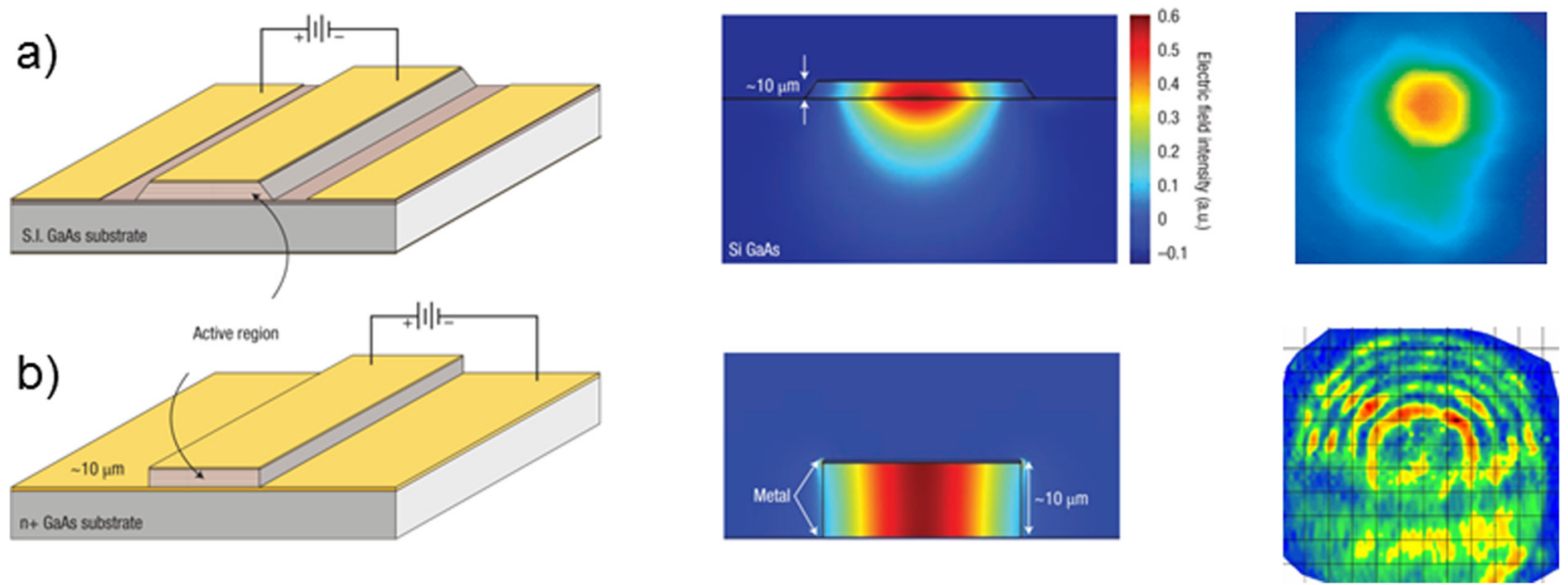

FIG. 3. (a) Surface-plasmon waveguide, mode distribution, and output beam profile (from left to right). (b) The same for a QCL with a metal-metal waveguide. Adapted with permission from B. S. Williams, Nat. Photonics 1, 517 (2007). Copyright 2007 Springer Nature; Adam et al., Appl. Phys. Lett. 88, 151105 (2006). Copyright 2006 AIP Publishing LLC.

such effects are observed for many active-region types. While lasing might still be possible, spectroscopy is strongly impeded by the presence of such phenomena due to the inherently uncontrollable behavior, which is typically reflected by instabilities and jumps in the current-voltage dependence.

Many active-region designs involve phonon-assisted transitions: If the energy spacing between two subband levels is sufficiently large, electrons are very efficiently transferred into the lower level by emitting a longitudinal-optical phonon. This can be exploited to inject electrons into the upper laser level or extract them from the lower laser level. However, it implies also an additional voltage drop for each period due to the phonon energy $(\sim 36 \mathrm{meV}$ in $\mathrm{GaAs}$ ), which results in a large operational voltage and consequently a large electrical heating. While most temperature and power records are based on so-called resonant-phonon designs, ${ }^{30,31}$ they have gained only limited relevance for high-resolution spectroscopy due to the limitation to pulsed operation and/or the huge driving powers for continuous-wave operation. More successful with respect to spectroscopy are bound-to-continuum designs, for which the depopulation of the lower laser level is provided by several closely spaced subband states. ${ }^{32}$ As compared to resonant-phonon designs, the operating voltage is much smaller and in consequence also the electrical driving power. Another successful strategy has been to combine the advantages of resonant-phonon scattering with a bound-to-continuum approach. The smaller operating voltage as compared to pure resonant-phonon designs significantly facilitates continuous-wave $(\mathrm{cw})$ operation in small cryocoolers. ${ }^{33-37}$ Typical $\mathrm{cW}$ operation temperatures in such coolers are $40-60 \mathrm{~K}$ with a record temperature close to $130 \mathrm{~K}^{38}$

Besides the active region, QCLs rely on a resonator, which confines the optical mode (Fig. 3). This implies an optical waveguide and feedback elements, which are in the simplest case the cleaved facets of a Fabry-Pérot laser ridge. Two major waveguide types have been realized for $\mathrm{THz}$ QCLs. These are single-plasmon (or semiinsulating surface plasmon, SP) and metal-metal (MM) waveguides.
For SP waveguides, the optical mode is vertically confined by a top metal layer and a high-doped bottom contact layer underneath the active region. The evanescent field of the optical mode penetrates substantially into the substrate, which therefore has to be semiinsulating in order to provide low optical losses. For MM waveguides, the active region is sandwiched between two metal layers. This involves additional wafer-bonding and substrate-transfer steps during fabrication. The optical mode is confined between the top and the bottom metal layer. Due to the strong subwavelength confinement, the beam quality and output power of edge-emitting MM QCLs are worse in comparison with SP devices. However, MM QCLs have the advantage to operate at higher temperatures. While for SP devices, the laser feedback is either provided by a Fabry-Pérot cavity or a distributed feedback (DFB) grating, the strong optical confinement of the MM waveguide allows for a plethora of resonator designs. This has been a playground of QCL development in the recent decade, due to the many degrees of freedoms for the design. $^{40-42}$ In fact, many of the drawbacks concerning the beam profile of MM QCLs and resonant-phonon active regions were overcome by resonator designs, often with subwavelength lateral dimensions. With respect to spectroscopy, there is one major practical implication of such approaches: Due to resonator dimensions much smaller than the wavelength, the optical mode becomes literally squeezed out of the semiconductor with the consequence that the resonator frequency becomes affected by the environment. While one might not expect this to become an issue since QCLs are operated in vacuum, it turned out that the almost unavoidable adsorption of residual gas is sufficient to result in an unacceptable frequency drift with time. ${ }^{37,43}$ Only recently, drift effects were suppressed by embedding the resonator in a polymer. ${ }^{44}$

\section{B. Output power}

The output power of a QCL is an important figure-of-merit, although its significance for spectroscopy is less than for imaging 
applications. A record $\mathrm{cw}$ output power up to $230 \mathrm{~mW}$ has been obtained with a QCL operating at $3.1 \mathrm{THz}{ }^{45}$ The active medium is based on a bound-to-continuum design with resonant-phonon extraction, which is embedded in a SP resonator. It is worth noting that this laser has a beam profile close to a fundamental Gaussian. Similar output power $(138 \mathrm{~mW})$ has been demonstrated by another group ten years before with a QCL operating at $4.4 \mathrm{THz}^{30}$ It should be noted that both QCLs were operated at $10-15 \mathrm{~K}$ in order to generate such high output power. Nevertheless, at temperatures of a few tens of kelvins, which are accessible by mechanical coolers, the lasers still deliver several $10 \mathrm{~mW}$. Based on these results, one can expect that it is possible to achieve tens of $\mathrm{mW}$ with dedicated QCL designs at frequencies above $2 \mathrm{THz}$ and operating temperature accessible with compact cryocoolers. Comparing this performance with the requirements for spectroscopic applications, it becomes clear that output power is not a major limitation, possibly with the exception of photoacoustic spectroscopy. In any case, the output power always needs to be considered along with several other parameters, namely, line width, frequency stability, and beam profile.

\section{Frequency stabilization}

Linewidth and frequency stability determine the number of photons per spectral bin, also called brilliance. There are several approaches for frequency stabilization of a QCL. The foremost one is passive stabilization based on thermal and electrical bias control, because of its relative simplicity. Other stabilization techniques require a decent a priori frequency stability in order to perform adequately. Passive frequency stabilization relies on the fact that the QCL frequency changes with the temperature of the active medium (typically in the order of a few $100 \mathrm{MHz} / \mathrm{K}$ ) and with the driving current of the QCL (typically a few $\mathrm{MHz} / \mathrm{mA}$ ). Keeping the temperature and current stable at the level of $1 \mathrm{mK}$ and $1 \mu \mathrm{A}$ yields a frequency-stable operation of the QCL. This scheme has been implemented, for example, for the QCL LOs in the heterodyne spectrometers GREAT and upGREAT on board of SOFIA. A frequency stability of $1.6 \mathrm{MHz}$ was achieved. ${ }^{9}$

Active frequency stabilization requires some sort of reference. The first demonstration of a QCL, which has been frequency and phase-locked to a reference source, has been made by Betz et al. ${ }^{46}$ They locked the frequency of a 3-THz QCL to that of on optically pumped $\mathrm{THz}$ gas laser. The line shape of the QCL was found to be essentially Gaussian with a full width at half maximum (FWHM) of $65 \mathrm{kHz}$. Further improvements of this technique yielded a 3-4 kHz FWHM of a $2.5-\mathrm{THz}$ QCL. ${ }^{47}$ While these experiments proved the feasibility of frequency- and phase-locking of a QCL, it is not a very practical approach, because the gas laser is bulky and difficult to handle and it is a fixed frequency source.

A more practical approach for a frequency stabilization of a QCL is using a microwave oscillator as a reference. The output signal of the microwave oscillator is amplified and multiplied to obtain a frequency, which is close to the frequency of the QCL. Since microwave oscillators are available with $1 \mathrm{~Hz}$ linewidth and frequency multiplication increases both, the linewidth and the phase noise only by $20 \log (\mathrm{n})$ (n: multiplication factor), this approach provides a highly stable and precise reference frequency.
The first demonstration of this technique has been done with a 1.5-THz QCL and a multiplier THz source. The IF signal, which is generated by a superconducting HEB mixer, is downconverted to about $200 \mathrm{MHz}$ in order to drive a phase-locked loop (PLL) circuit, which controls the bias voltage of the QCL. A frequency stability of better than $100 \mathrm{~Hz}$ has been demonstrated. ${ }^{48}$ A major challenge in this approach is the generation of sufficient output power from the multiplier source as well as replacing the superconducting mixer with a mixer, which is capable of operation at ambient temperature. This can been solved by using a GaAs/AlAs superlattice diode, which generates the required harmonic from a solid-state $\mathrm{THz}$ source and which serves at the same time as a mixer for generating the IF signal from the QCL emission. With this technique, phaselocking of a 3.4-THz QCL was achieved with a linewidth of $48 \mathrm{kHz}$ (Fig. 4). ${ }^{49}$ An alternative device to generate powerful harmonics from a microwave source is a $\mathrm{THz}$ Schottky diode harmonic mixer. Such a mixer has been developed for $2.3-3.2 \mathrm{THz}$ operation. Its main part is a GaAs based $\mathrm{THz}$ circuit with an integrated diode. When this harmonic mixer is pumped by a $0.63 \mathrm{THz}$ multiplier

a)

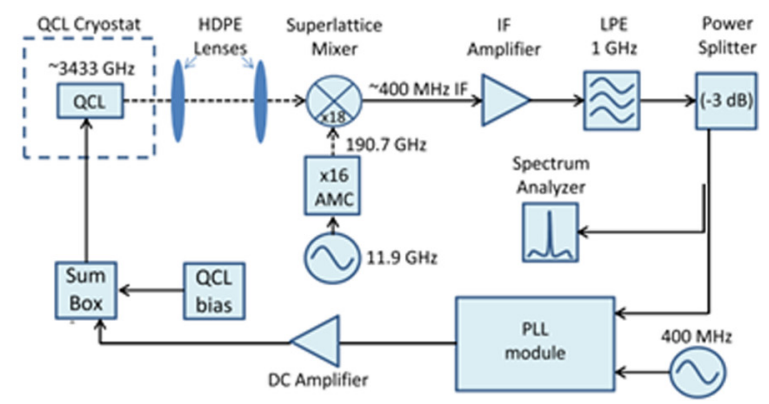

b)

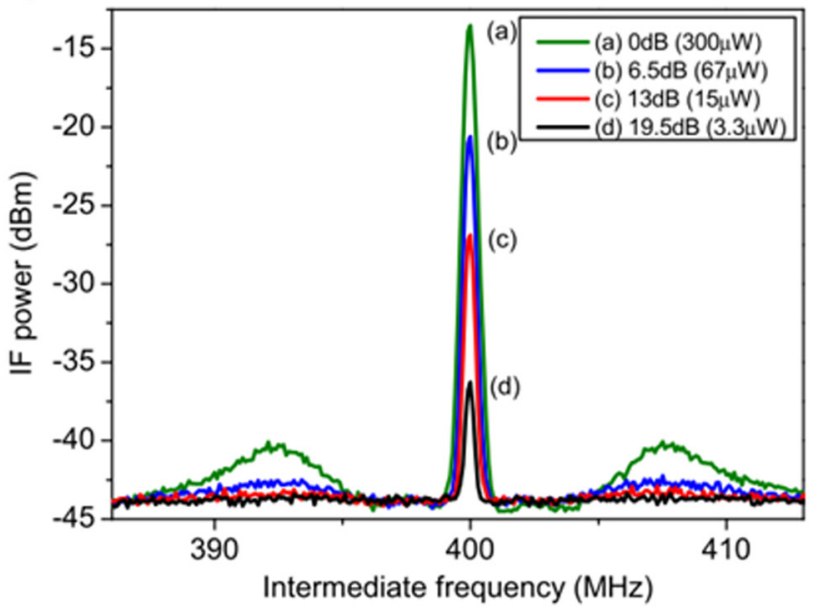

FIG. 4. (a) Phase-locking scheme with a microwave reference source. (b) Power spectrum of the IF signal for the phase-locked system for various optical attenuation of the QCL beam. Adapted with permission from Hayton et al., Appl. Phys. Lett. 103, 051115 (2013). Copyright 2013 AIP Publishing LLC. 
source and a 2.5-THz QCL, it generates the fourth harmonic of the multiplier signal, which, in turn, generates an IF signal with the QCL frequency, which is used for phase-locking of the QCL. With this approach, a beat signal with $1 \mathrm{~Hz}$ linewidth (FWHM) has been demonstrated. ${ }^{50}$

Instead of a microwave source, a $\mathrm{THz}$ frequency comb can be used as a stable reference for the QCL frequency. Phase-locking of a longitudinal mode of a $2.7-\mathrm{THz} \mathrm{QCL}$ to a $\mathrm{THz}$ frequency comb yields a beat-note signal with a bandwidth of $1 \mathrm{~Hz}$, which is generated by a HEB mixer. ${ }^{51}$ However, the absolute frequency uncertainty was much larger $(1 \mathrm{kHz})$, because the frequency comb was freerunning. Metrological-grade $\mathrm{THz}$ spectroscopy of a $\mathrm{CH}_{3} \mathrm{OH}$ transition has been demonstrated with a $2.5-\mathrm{THz} \mathrm{QCL}$, which has been phase-locked to a $\mathrm{THz}$ frequency comb, which, in turn, has been referenced to a Cs frequency standard. ${ }^{7}$ By tuning the repetition rate of the frequency comb, its frequency as well as the frequency of the
QCL was swept across a $\mathrm{CH}_{3} \mathrm{OH}$ transition. The absolute frequency uncertainty of the QCL was a few parts in $10^{-11}$ and the line center of the $\mathrm{CH}_{3} \mathrm{OH}$ transition has been determined with an uncertainty of $10^{-9}$ (Fig. 5). Instead of using an external mixer for generating the beat note, it is also possible to injection-lock the QCL to a frequency comb. A photomixer generates a $\mathrm{THz}$ reference signal from two $1.55-\mu \mathrm{m}$ semiconductor lasers, which are locked to a near-infrared frequency comb. The comb line spacing is referenced to a frequency stabilized microwave source. By this means, the frequency of the QCL is locked to an integer harmonic of the microwave reference. ${ }^{52}$

In analogy to atomic clocks, one can use certain molecular transitions with well-defined frequency. In this approach, the absolute frequency accuracy is given by the precision of the knowledge of molecular transition frequency. This can be quite different depending on the molecular species. In the case of $\mathrm{CH}_{3} \mathrm{OH}$, for example, it is typically about $1 \mathrm{MHz},{ }^{53}$ which corresponds to a relative uncertainty

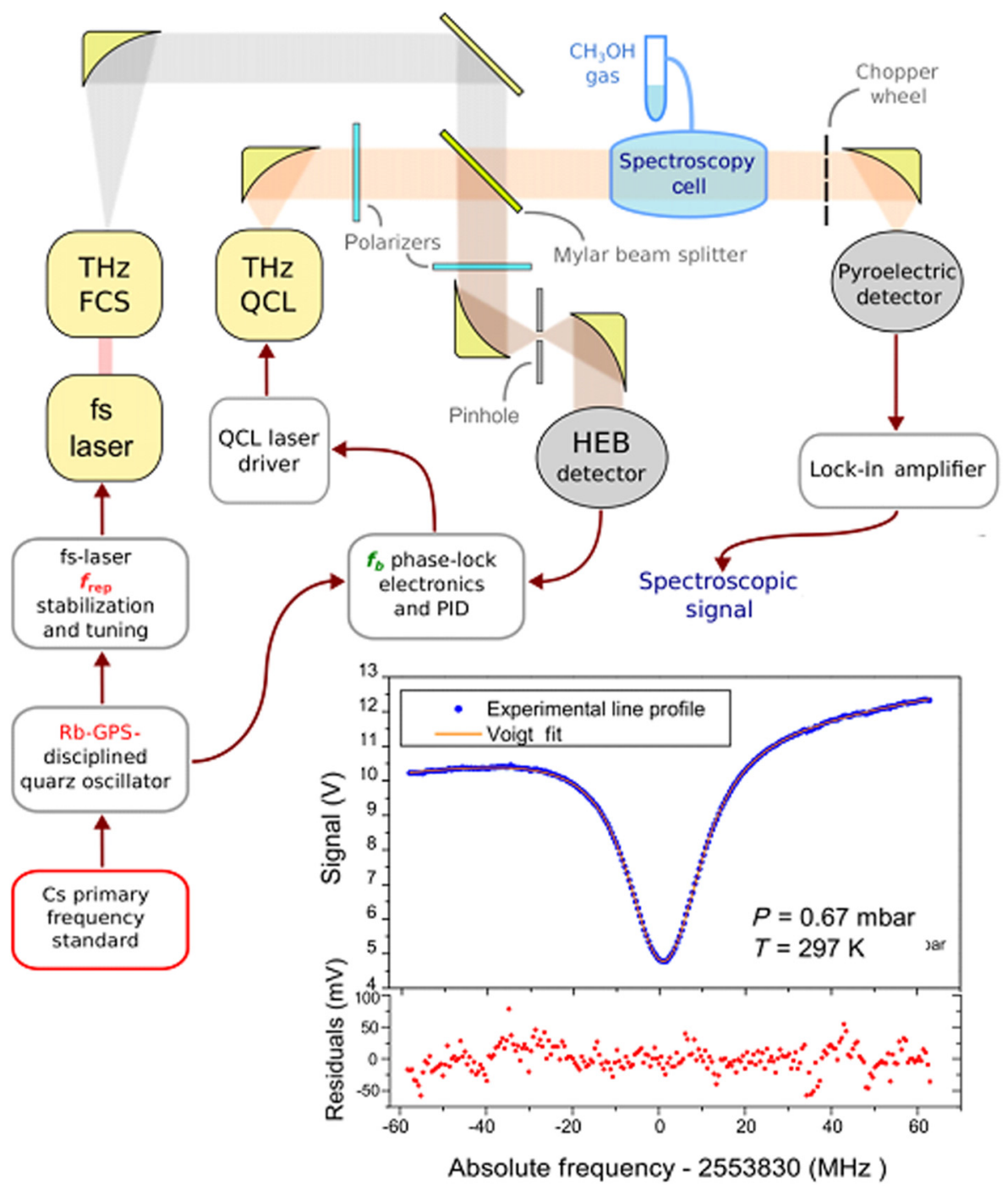

FIG. 5. Experimental setup of a $\mathrm{THz}$ spectrometer with $\mathrm{QCL}$. The frequency of the QCL is stabilized to a frequency comb. Note that the frequency of the comb is traced to a primary Cs frequency standard. The inset shows an absorption line of $\mathrm{CH}_{3} \mathrm{OH}$ measured with this spectrometer and the corresponding residuals relative to a fit with a Voigt line profile. Adapted from Bartalini et al., Phys. Rev. X 4, 021006 (2014). Copyright 2014 Author(s), licensed under a Creative Commons Attribution 4.0 License. 
of $10^{-6}-10^{-7}$, while for $\mathrm{CO}$, it is less than $10 \mathrm{kHz}^{54}$ (relative accuracy $\sim 10^{-8}$ ). A frequency accuracy of $1 \mathrm{MHz}$ and a linewidth of $51 \mathrm{kHz}$ were demonstrated by locking the QCL frequency to a rotational transition of $\mathrm{CH}_{3} \mathrm{OH}$ (Fig. 6). ${ }^{55,56} \mathrm{~A}$ much better precision, typically an order of magnitude, can be achieved when the Lamb-dip of a transition is used for frequency stabilization. However, so far, this has not been demonstrated with a THz QCL, and for certain applications such as spaceborne missions, the gas cell is a rather challenging device.

\section{Frequency tuning}

As for other semiconductor lasers, frequency tuning of $\mathrm{THz}$ QCLs is typically realized by varying the driving current and/or the
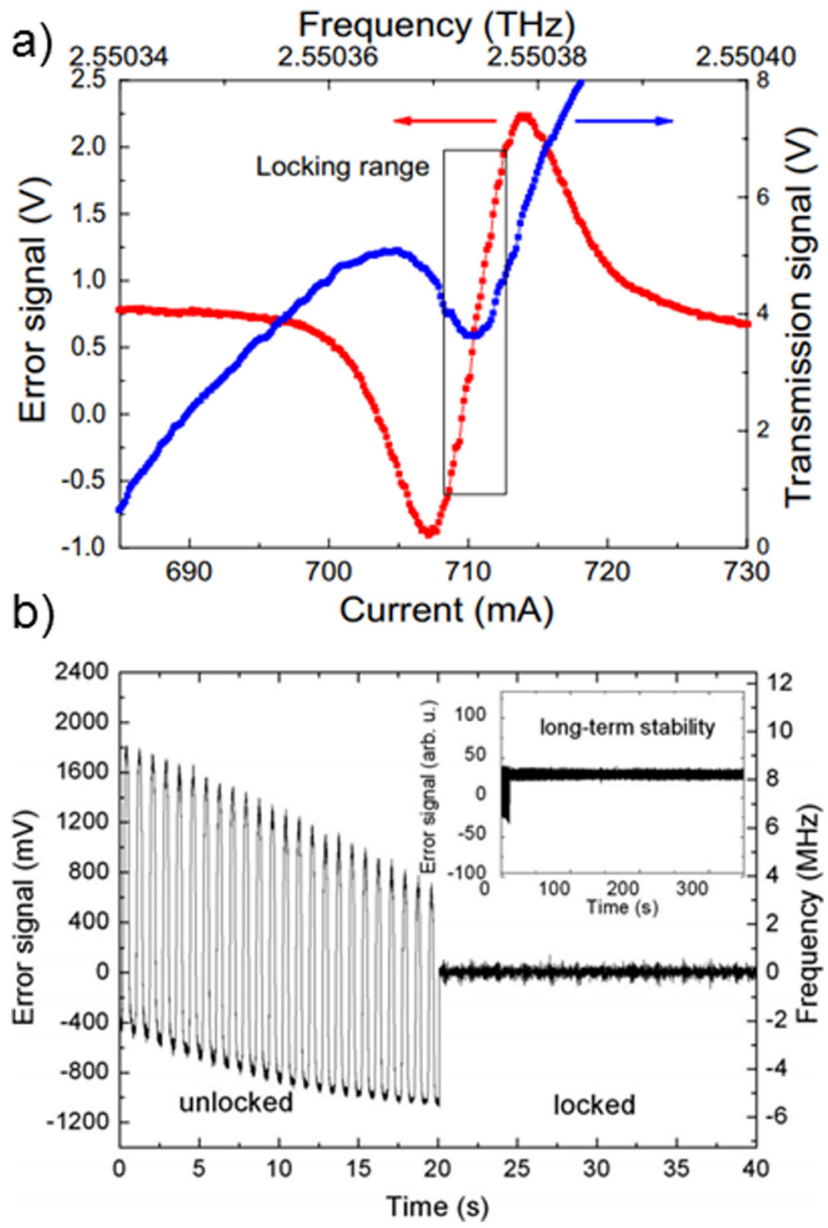

FIG. 6. (a) Error signal and transmission signal of a frequency stabilization with a rotational transition of $\mathrm{CH}_{3} \mathrm{OH}$ at $2.55 \mathrm{THz}$ as a reference. (b) Frequency variation of the QCL in the unlocked state (first $20 \mathrm{~s}$ ) and in the locked state. The frequency variations in the unlocked state are due to temperature fluctuations of the cryocooler. In the locked state, the linewidth of the QCL is $300 \mathrm{kHz}$ (FWHM). Adapted with permission from Richter et al., Appl. Phys. Lett. 96, 071112 (2010). Copyright 2010 AIP Publishing LLC. heat sink temperature. However, the obtainable tuning range by this means is usually well below $10 \mathrm{GHz}$. Hence, there has been a strong motivation for developing methods, which enhance the frequency tuning capability of $\mathrm{THz}$ QCLs. Several techniques for enhanced continuous frequency tuning were demonstrated during recent years. Among them are external optical cavities ${ }^{57-59}$ with up to $50 \mathrm{GHz}$ of continuous tuning, ${ }^{59}$ micro-optomechanical cavities ${ }^{60,61}$ with up to $240 \mathrm{GHz}$, ${ }^{61}$ electronic frequency tuning in multiterminal and multisection QCLs ${ }^{62-65}$ with up to $19 \mathrm{GHz},{ }^{63}$ as well as tuning by gas adsorption with up to $25 \mathrm{GHz} .{ }^{43}$ A small frequency tuning of a few hundred $\mathrm{MHz}$ is obtainable by external optical feedback, which is already sufficient to do spectroscopy of close-by molecular transitions. ${ }^{66}$ However, so far, none of these approaches has been established for high-resolution spectroscopy, which might be caused by complicated setups and device fabrication procedures, or by noncontinuous or nonreproducible frequency tuning characteristics. Using unfocused near-infrared illumination, Hempel et al. demonstrated a laser-induced frequency tuning (LIFT) of several $\mathrm{GHz}$ for $\mathrm{THz}$ QCLs with a SP waveguide. $^{67,68}$ Recently, a tuning range of $40 \mathrm{GHz}$ was demonstrated and the feasibility of this approach for high-resolution molecular spectroscopy was shown ${ }^{69}$ (Fig. 7). It was found that by wellcontrolled, focused near-infrared excitation of the substrate just below the active medium, a frequency coverage exceeding ten times the tuning by current is obtainable. The tuning mechanism relies on the generation of a nonthermal electron-hole plasma in the vicinity of the back facet of edge-emitting devices, which changes the optical cavity length and consequently the mode frequency. The method is rather straight forward to implement and can be readily applied to a large class of THz QCLs.

\section{E. Beam profile}

The beam profile is important, because for most applications, only the power that is available in the fundamental Gaussian mode is useful. Power that is in higher order modes often leads to artifacts in the spectrum such as standing waves or it causes optical feedback and frequency instabilities. The beam profile of a QCL depends strongly of the type of a waveguide. The beam profile of a QCL with a SP waveguide has contributions from the laser ridge and from the substrate [Fig. 3(a)]. ${ }^{70,71}$ With such a laser, an $\mathrm{M}^{2}$ value of less than 1.2 has been demonstrated ${ }^{72}$ by using a dedicated lens and some spatial filtering close to the outcoupling facet of the QCL. The beam pattern of a QCL with a MM waveguide is much more divergent and a main lobe is not observable. Instead, a pronounced ring-like pattern exists, which results from the far field interference of the coherent radiation emitted by all facets of the laser ridge [Fig. 3(b)]. ${ }^{39}$ Several methods have been developed in order to improve the beam patterns of QCLs, in particular, QCLs with MM waveguides. Examples are a silicon lens, a Winston cone, a hollow dielectric waveguide or a horn antenna mounted to the outcoupling facet of the QCL, ${ }^{47,73-75}$ second- or third-order distributed feedback (DFB) gratings, ${ }^{40,76,77}$ photonic bandgap structures, ${ }^{78,79}$ or a phase-locked array of surface-emitting QCLs. ${ }^{80}$ However, none of these approaches has resulted in an improved beam profile when compared with QCLs with a SP resonator and beam shaping optics. 

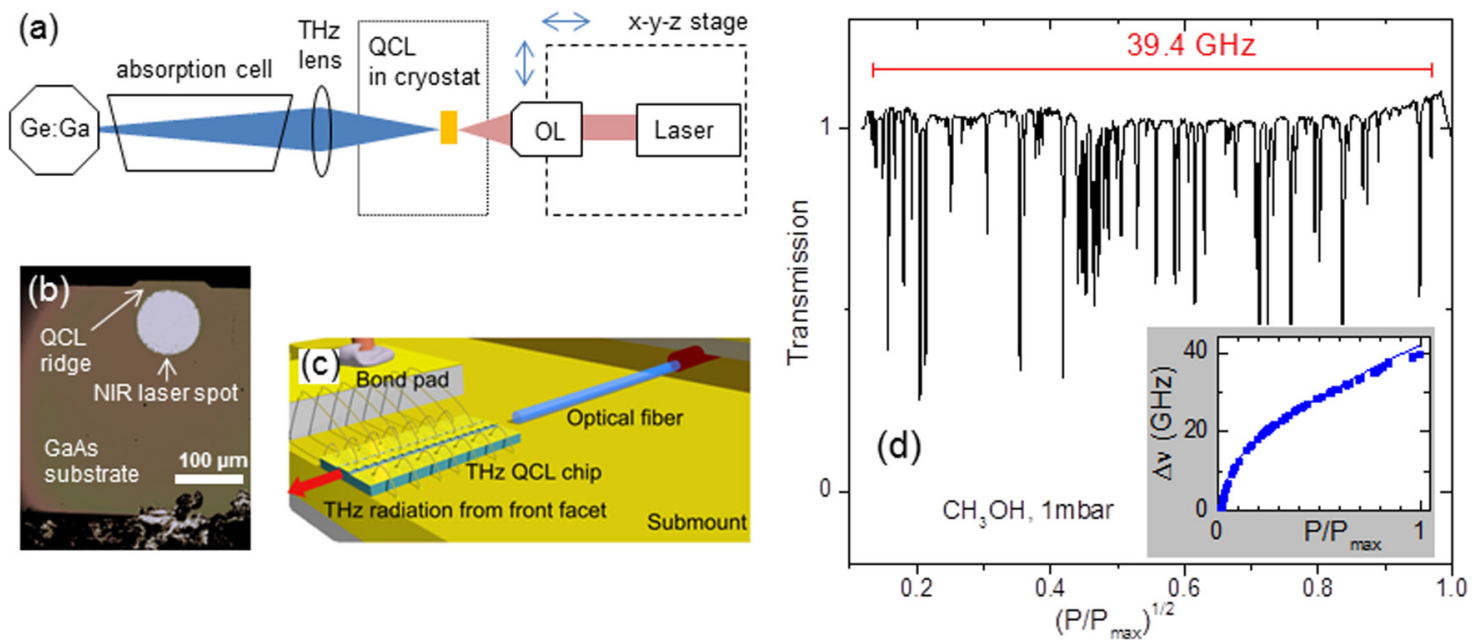

FIG. 7. (a) Experimental configuration for absorption spectroscopy based on LIFT with a near-infrared laser and a microscope objective lens (OL). Adapted with permission from Alam et al., Opt. Express 27, 5420 (2019). Copyright 2019 OSA. (b) Near-infrared laser spot at the rear facet of the QCL. Adapted with permission from Alam et al., Opt. Express 27, 5420 (2019). Copyright 2019 OSA. (c) Alternative LIFT configuration with an optical fiber. Adapted with permission from Hagelschuer et al., Opt. Express 25, 30203 (2017). Copyright 2016 AIP Publishing LLC. (d) LIFT-based molecular absorption spectrum measured with the setup shown in (a) and covering approximately $40 \mathrm{GHz}$. Inset: QCL frequency vs illumination power, showing a square-root dependence typical for LIFT.

Recently, a vertical-external-cavity surface-emitting laser (VECSEL) based on a THz QCL was demonstrated. ${ }^{81,82}$ Because the cavity of a VECSEL is designed to support only the fundamental Gaussian mode, a very high beam quality can be obtained. However, for a $\mathrm{THz} \mathrm{QCL}$, this concept cannot be implemented easily, because the electric field of the QCL is polarized perpendicular to the plane of the quantum wells and barriers. This obstacle has been overcome by an amplifying metasurface reflector, which consists of an array of parallel metal-metal waveguides spaced less than the free-space wavelength. Each waveguide is filled with the active medium. The width of the waveguides and their thickness is much smaller than the laser wavelength and each waveguide has a low Q-factor. However, when the array is placed in a high-Q external cavity, it provides gain and lasing can occur. The beam profile is determined by the properties of the external cavity formed by the metasurface reflector and the external mirror. With a hemispherical externalcavity Gaussian beam with an $\mathrm{M}^{2}$ beam parameter as low as 1.3 has been demonstrated. ${ }^{82}$

\section{ABSORPTION SPECTROSCOPY}

\section{A. Linear absorption spectroscopy}

The first demonstration of high-resolution molecular spectroscopy with a THz QCL has been published in 2006 (Fig. 8). ${ }^{83}$ The spectrometer was based on a $2.5-\mathrm{THz}$ QCL with a DFB resonator and a liquid-helium cooled Ge:Ga photodetector. The frequency was tuned by changing the QCL driving current and the absolute frequency was determined by mixing its radiation with that from an optically pumped $\mathrm{THz}$ gas laser. The frequency of the $\mathrm{v}_{\mathrm{t}}=2-1 \quad\left(\mathrm{~J}_{\mathrm{K}}\right) 2_{02}-2_{13}$ rotational transition of $\mathrm{CH}_{3} \mathrm{OH}$ has been determined to be $2.519112(1) \mathrm{THz}$. The precision of approximately $1 \mathrm{MHz}$ was limited by the accuracy of the gas laser frequency. This measurement is in excellent agreement with a measurement of the same transition with a multiplier source, which yields $2.519109986(50) \mathrm{THz}^{19}$ and with the calculated frequency of $2.519110022(152) \mathrm{THz}^{84}$ The relative frequency accuracy of the QCL-based spectrometer is even better and allows determining the pressure broadening and the pressure shift of the transition with an accuracy of $\sim 2 \mathrm{kHz}$.

In order to eliminate external fluctuations such as QCL temperature instabilities, a differential acquisition technique can be employed. In such a scheme, the radiation from the QCL is diverted into two optical paths: One path contains the absorption cell and the other one is without an absorption cell. The signals of both optical paths are detected with identical detectors and the detector outputs are balanced onto a differential amplifier. In combination with frequency modulation, this approach has been shown to yield a four times larger SNR than direct absorption spectroscopy without differential detection.

Wavelength or frequency modulation schemes have been implemented in QCL absorption spectrometers for improving their sensitivity (Fig. 8). In both techniques, the frequency of the QCL is modulated, for example, by modulating its driving current. The laser radiation is detected with the modulation frequency as a reference. The term wavelength modulation is used when the amplitude of the modulation frequency is smaller than the width of the investigated absorption line. When it is larger than the linewidth, the technique is called frequency modulation. The advantage of wavelength and frequency modulation is that limitations by low-frequency noise of the laser can be overcome if the modulation frequency is sufficiently large. Amplitude as well as wavelength/frequency modulation schemes have been realized with 

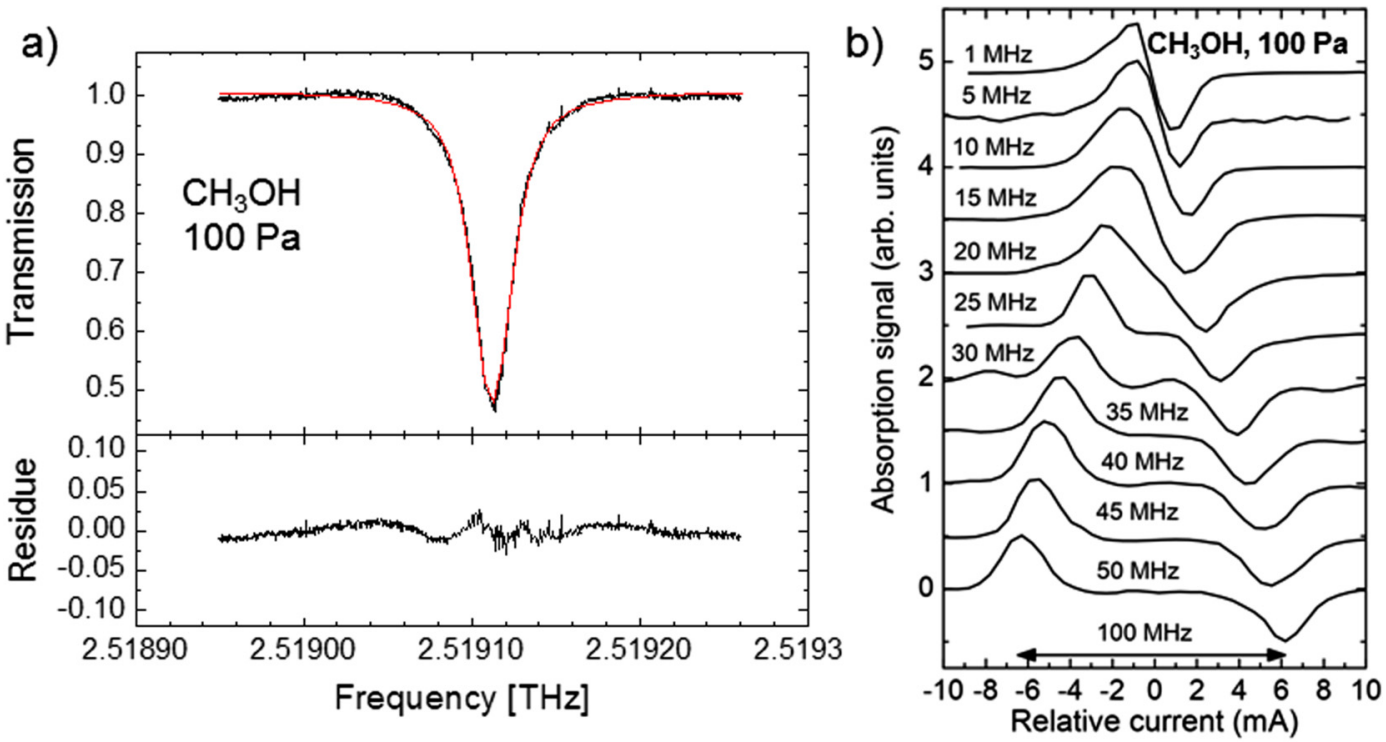

FIG. 8. (a) Direct absorption spectrum of $\mathrm{CH}_{3} \mathrm{OH}$ at $2.5 \mathrm{THz}$. Adapted with permission from Hübers et al., Appl. Phys. Lett. 89, 061115 (2006). Copyright 2006 AIP Publishing LLC. (b) 1f-absorption spectrum of $\mathrm{CH}_{3} \mathrm{OH}$ at $3.1 \mathrm{THz}$. The figure shows the transition from wavelength modulation (top) to frequency modulation (bottom). The frequency at each spectrum is the modulation frequency. Adapted with permission from Eichholz et al., Opt. Express 21, 32199 (2013). Copyright 2019 OSA.

QCL absorption spectrometers and it was shown that with frequency/ wavelength modulation, the baseline slope due to the scanning laser can be removed and the SNR can be significantly improved because of the narrow detection bandwidth of the lock-in detection., ${ }^{5,73,83}$ If a single-mode QCL is used, the frequency coverage of the spectrometer is limited to a few GHz. It can be extended by using a multimode QCL in combination with a monochromator. Coarse frequency setting is done by selecting a mode of the QCL with the monochromator. Fine frequency tuning is done by changing the driving current or the temperature of the QCL. If a multipixel detector is used instead of a single pixel, the absorption spectra obtained with each mode can be measured simultaneously. ${ }^{85}$ Also, LIFT has been shown to improve the frequency coverage significantly up to $40 \mathrm{GHz}$ [Fig. 7(d)]. ${ }^{68}$ The combination of LIFT with a multimode QCL, with a mode separation as large as the LIFT tuning range, and a monochromator may increase the frequency coverage even further.

Besides laser absorption spectroscopy, THz QCLs can also be employed as sources in high-resolution Fourier-transform spectrometers. Multimode devices can act as a high-brilliance alternative to the conventional globar source. ${ }^{86}$ Multimode devices have also gained a great attraction in recent years due to frequency-comb operation and the closely related dual-comb spectroscopy. ${ }^{87-89}$ In particular, the large spectral coverage of frequency combs is important. A cw THz QCL that covers one octave has already been demonstrated. ${ }^{90}$ A first spectroscopic application of THz QCL combs has been demonstrated as well. ${ }^{91}$ However, these are still restricted to low-resolution results, since the very limited frequency tuning in comb regimes does not allow for a continuous spectral coverage.

For real-world applications, liquid-helium cooled detectors are not an option. A sensitive technique for gas-phase detection of molecules, which does not require a cryogenically cooled detector, is photoacoustic spectroscopy. In its most sensitive and compact implementation, called quartz-enhanced photoacoustic spectroscopy (QEPAS), spectrometers with detection limits as low as several ten parts per trillion have been demonstrated at near-infrared and midinfrared frequencies. The core element of such a spectrometer is a quartz tuning fork (QTF), which is placed inside the absorption cell. The QTF has two arms that oscillate when a sinusoidal voltage is applied at its resonance frequency. In THz QEPAS, the frequency of the QCL is tuned across a rotational transition of the gas between the arms of the QTF (Fig. 9). ${ }^{6}$ Upon absorption, the gas is heated and the resonance frequency of the QTF changes. This changes the voltage at the QTF terminals, which is detected by means of a lock-in amplifier. Using a 3.9-THz QCL, a minimum detection limit of $160 \mathrm{ppb}$ $\mathrm{CH}_{3} \mathrm{OH}$ in $30 \mathrm{~s}$ integration time has been demonstrated. ${ }^{92}$

All spectroscopy techniques discussed so far rely on external detectors, which make the spectrometers more complicated and expensive, in particular, when the detector is cooled by liquid helium. This can be overcome by using optical feedback, which changes the QCL frequency as well as its terminal voltage. In order to perform spectroscopic measurements, the absorption cell is placed between the QCL and a mirror, which reflects the radiation back into the QCL. The frequency of the QCL is tuned by changing the position of the reflecting mirror. When the frequency coincides with a molecular absorption line, the optical feedback is reduced and the terminal voltage of the QCL changes. This technique has been demonstrated with a mixture of $\mathrm{D}_{2} \mathrm{O}$ and $\mathrm{CH}_{3} \mathrm{OD}$. Fast measurement times on the order of $10 \mathrm{~ms}$ per spectrum and real-time measurements of gas concentrations with a rate of $100 \mathrm{~Hz}$ have been demonstrated. ${ }^{66}$ This is close to the performance, which can be achieved 
a)

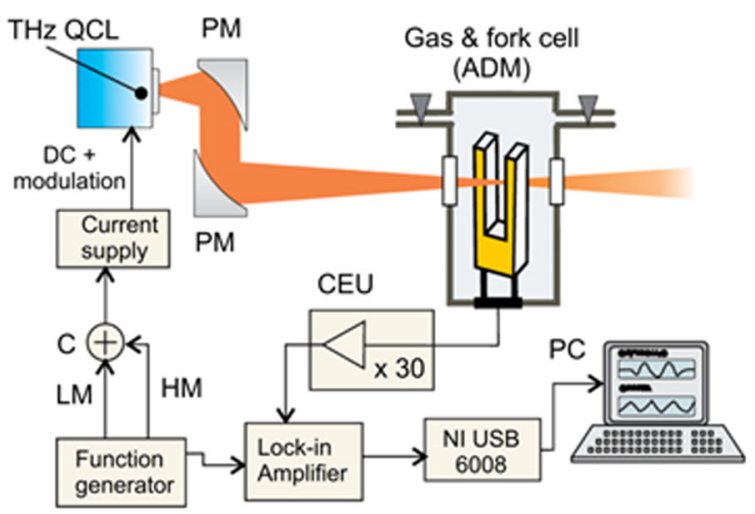

b)

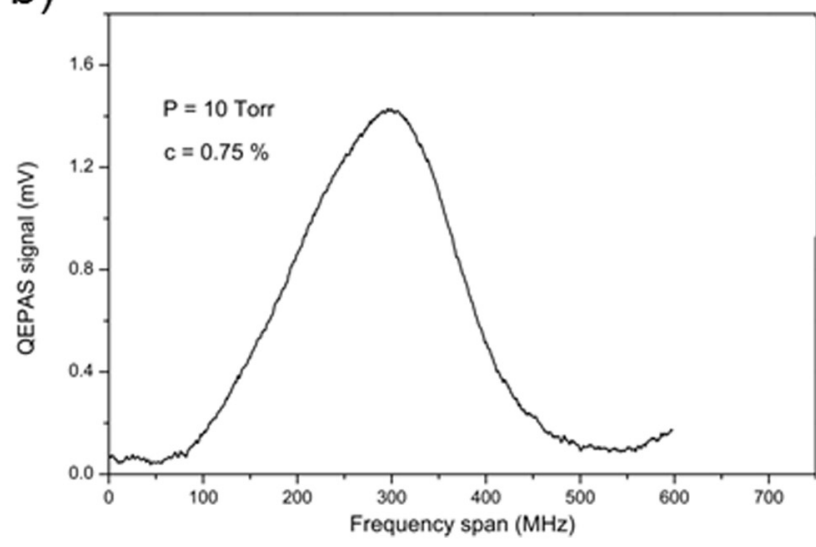

FIG. 9. (a) Experimental setup of a photoacoustic spectrometer with $\mathrm{THz} Q \mathrm{QLL}$. Adapted with permission from Borri et al., Appl. Phys. Lett. 103, 021105 (2013). Copyright 2013 AIP Publishing LLC. (b) Spectrum of $0.75 \% \mathrm{CH}_{3} \mathrm{OH}$ in $\mathrm{N}_{2}$ at 10 Torr. Adapted with permission from Borri et al., Appl. Phys. Lett. 103, 021105 (2013). Copyright 2013 AIP Publishing LLC

with a Ge:Ga detector. While the implementation of this approach is straightforward and its sensitivity is excellent and certainly will be improved in the future, the drawback is that the frequency depends on the strength of the optical feedback, i.e., on the strength of the absorption. Therefore, precise frequency measurements as well as line shape measurements are difficult and the method is more suited for sensing of molecules rather than for precision spectroscopy.

A far-reaching goal is the combination of high-resolution spectroscopy with real-time imaging. Such measurements provide information on the spatial distribution of molecules, which is relevant, for example, for monitoring chemical reactions. In principle, this can be done by expanding the beam of the QCL that illuminates the whole cross section of the absorption cell and using a $\mathrm{THz}$ camera behind the cell. However, in such a configuration, the power per pixel is small and the sensitivity of $\mathrm{THz}$ cameras is not as good as that of single pixel detectors. This reduces the sensitivity of such a spectrometer significantly. An alternative approach is to use a fast scanning mirror and a sensitive single pixel detector. ${ }^{93}$ Such a spectrometer has been realized, and spectral images of the distribution of $\mathrm{CH}_{3} \mathrm{OH}$ in a gas cell have been measured. Each image consists of 1350 pixels and each pixel is the average of several $200-\mathrm{MHz}$ wide spectra. The spatial resolution is $1 \mathrm{~mm}$ and the spectral resolution is $2 \mathrm{MHz}$. The images provide information on the spatial distribution of $\mathrm{CH}_{3} \mathrm{OH}$ in the gas cell as well as on its pressure. The setup allows for the observation of gas dynamics and leak detection as demonstrated with a $\mathrm{CH}_{3} \mathrm{OH}$-filled $\mathrm{THz}$-transparent tube. The system proves the feasibility of fast simultaneous high-resolution spectroscopy and high-resolution imaging at $\mathrm{THz}$ frequencies.

\section{B. Nonlinear (saturation) spectroscopy}

Limitations in frequency resolution and frequency accuracy caused by Doppler broadening can be overcome by nonlinear spectroscopy techniques, namely, saturation spectroscopy or Lamb-dip spectroscopy. There are a few requirements regarding the observation of a Lamb-dip: (i) The line profile of the transition should be broadened only by the Doppler effect, i.e., the pressure in the absorption cell has to be very low (in the order of $0.1 \mathrm{~Pa}$ ). (ii) The power from the source should be large enough to partially saturate the transition. (iii) The experimental setup should provide a pump-probe configuration, realized, for example, by overlapping beams in opposite directions. For a $\mathrm{THz}$ source, these conditions are not easily fulfilled. For example, it has taken $17 \mathrm{~h}$ to observe a Lamb-dip of a $\mathrm{CH}_{3} \mathrm{OH}$ transition at $2.5 \mathrm{THz}$ with a multiplier source. The signal-to-noise ratio of this measurement was four. ${ }^{19}$ In 2015, evidence of saturation effects in a rotational transition of $\mathrm{CH}_{3} \mathrm{OH}$ by using a $2.5-\mathrm{QCL}^{94}$ and in 2018, the first demonstration of Lamb-dip spectroscopy with a THz QCL has been published. Wienold et al. observed a Lamb-dip in $\mathrm{HDO}$ using a free-running, single-mode QCL at $3.3 \mathrm{THz}$ (Fig. 10). ${ }^{8}$ The observed Lamb-dip has a sub-Doppler linewidth of $170 \mathrm{kHz}$, which is approximately three times larger than the theoretical limit given by transit time broadening, i.e., the beam waist of the QCL. It should be noted that an SNR of about 10 was achieved within a measurement time of less than $200 \mathrm{~ms}$.

With Lamb-dip stabilization, an absolute frequency accuracy in the order of a few $\mathrm{kHz}$ is possible (relative uncertainty $10^{-8}$ ). Improvements beyond that limit become feasible when approaching the Lamb-Dicke regime where the molecular motion along the spectroscopy beam direction is much smaller than the wavelength of the radiation. This has been demonstrated with a $\mathrm{THz}$ multiplier source whose frequency is locked to a hydrogen maser yielding a linewidth of less than $10 \mathrm{~Hz}$ at $1.3 \mathrm{THz}{ }^{20}$ In order to reduce the molecular motion, $\mathrm{HD}^{+}$ions were trapped and crystallized in a linear quadrupole trap. With this setup, an absolute linewidth of $1.3 \mathrm{kHz}$ (fractional linewidth: $1 \times 10^{-9}$ ) of a $\mathrm{HD}^{+}$transition at $1.3 \mathrm{THz}$ was observed. Obviously, an extremely narrow linewidth is essential for the observation of such narrow spectral lines. QCLs have been shown to have an intrinsic linewidth as narrow as $90 \mathrm{~Hz} .{ }^{95}$ Therefore, they are in principle suitable for $\mathrm{THz}$ spectroscopy in the Lamb-Dicke regime, provided that stabilization techniques can be implemented, which allow for reaching the ultimate linewidth of a QCL.

\section{HETERODYNE SPECTROSCOPY}

Soon after the first demonstration of a THz QCL in 2002, it was realized that this type of laser has the potential to become the 
(a)

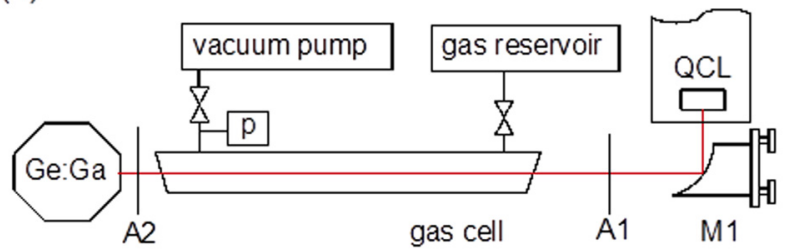

(b)

Frequency $(\mathrm{MHz})-3311.835 \mathrm{GHz}$

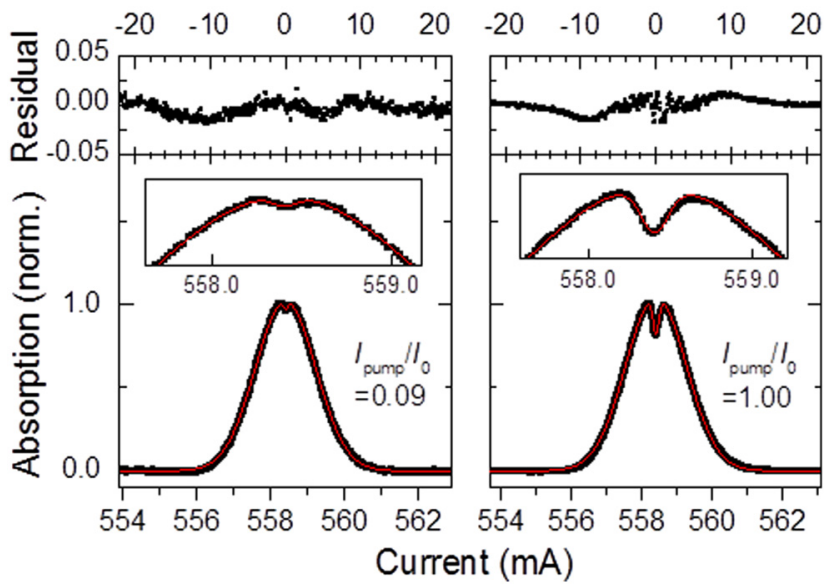

FIG. 10. (a) Experimental setup for Lamb-dip spectroscopy. (b) Lamb-dip spectra of $\mathrm{CH}_{3} \mathrm{OH}$ at $3.3 \mathrm{THz}$ measured at different pump powers and $2.5 \mu \mathrm{bar}$ pressure. The red line is a model calculation. Adapted with permission from Wienold et al., Opt. Express 26, 6692 (2018). Copyright 2018 OSA

long sought after LO for frequencies in the upper $\mathrm{THz}$ region, which are not accessible with multiplier sources. In 2005, the first noise temperatures were measured with a $\mathrm{THz} \mathrm{QCL}$ as $\mathrm{LO}$ and a HEB mixer. ${ }^{70,96}$ In one case, the QCL had a MM waveguide and was cooled with liquid helium ${ }^{96}$ and in the other case, the QCL had a SP waveguide and was mounted in a mechanical cryocooler. ${ }^{70}$ Most importantly, it was shown that the noise temperature measured with a QCL LO is the same as measured with an optically pumped gas laser LO. ${ }^{70}$ This demonstrated that the QCL does not add extra noise to the mixer. In the following years, major milestones were the demonstration of an integrated $2.5-\mathrm{THz}$ heterodyne spectrometer with a QCL LO and a HEB mixer mounted in a pulse tube cooler, ${ }^{72}$ the demonstration that a QCL, which fulfills all the requirements for a $\mathrm{LO}$, can be operated in a small cryocooler, ${ }^{97}$ and the laboratory measurement of molecular spectra with a QCL-based heterodyne spectrometer. ${ }^{98}$ An important issue when pumping a HEB mixer is the power stability of the QCL. Since the HEB is a bolometer, its sensitivity degrades with LO power instabilities and drift. For a QCL power, stabilization by current or temperature is not an option because this changes its frequency as well. An independent method is using a fast optical attenuator, which is placed between the QCL and the HEB. By using the direct detection output of the HEB mixer in combination with a proportional-integral-differential loop, a significant improvement has been achieved. ${ }^{56}$ Obviously, other important milestones are the various frequency stabilization schemes and beam shaping techniques, which are described in Sec. III.

In 2013, the first QCL was developed, which complies with all requirements regarding a $\mathrm{LO}$ for the observation of the OI fine structure line at $4.7 \mathrm{THz}$ with SOFIA; namely, it provides the exact frequency and sufficient frequency coverage. The QCL has an excellent beam profile and its output power across the whole frequency range is sufficient for pumping the mixer with ample margin. ${ }^{35}$ This paved the way for the first QCL LO for a real instrument, namely, the LO for the GREAT heterodyne spectrometer on board of SOFIA in 2014 (Fig. 11). ${ }^{9}$ The QCL has a SP waveguide and first-order lateral DFB grating, ${ }^{99}$ which yields single-mode emission with a precisely defined LO frequency. Frequency tuning is done by changing its driving current or its temperature. Frequency stability is ensured by highly stable current and temperature. The absolute frequency is determined from a current-temperature-frequency map, which is generated from heterodyne spectra of $\mathrm{CH}_{3} \mathrm{OH}$ measured at different current and temperature settings. Any possible frequency drift during operation on SOFIA can be monitored and

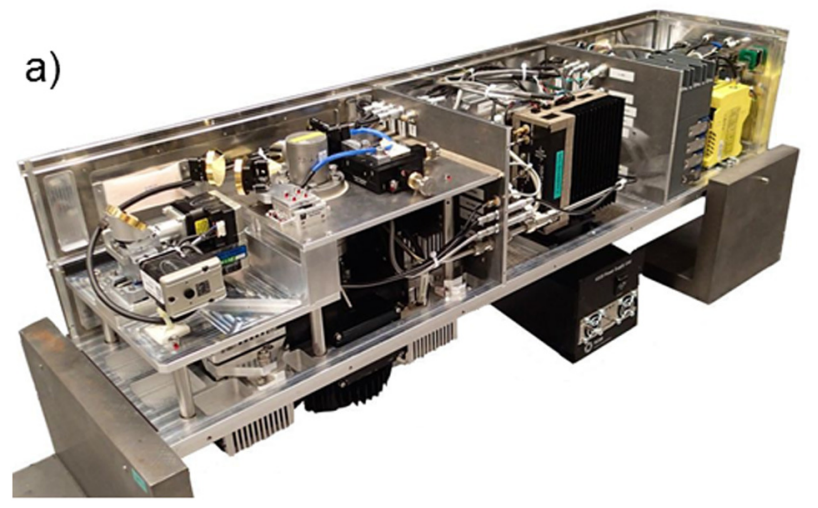

b)

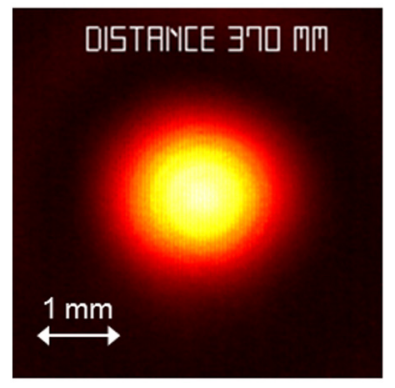

c)

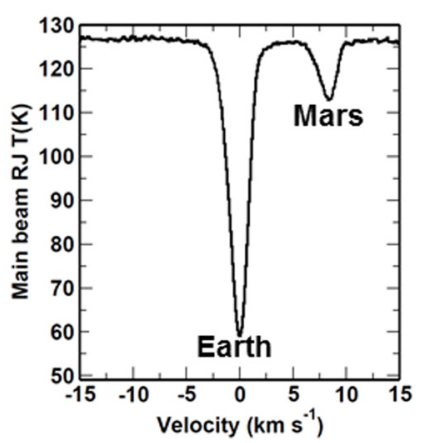

FIG. 11. (a) 4.7-THz LO for the upGREAT heterodyne spectrometer on board of SOFIA. (b) Beam profile of the QCL LO. (c) Spectrum of the OI transition at 4.7 THz in the atmospheres of Earth and Mars. The frequency scale is given in velocity units according to the Doppler shift. Adapted with permission from Rezac et al., Astron. Astrophys. 580, L10 (2015). Copyright 2015 ESO. 
corrected, because the OI line, which originates from the mesosphere and lower thermosphere of the Earth, is measured simultaneously with each astronomical spectrum. The QCL is mounted in a compact mechanical cryocooler, which in turn is mounted in a dedicated LO box [Fig. 11(a)]. This box contains the beam shaping optics, the power supply, and the control electronics. The actual version delivers up to $2 \mathrm{~mW}$ power in a Gaussian beam. It covers the frequency span from $-2 \mathrm{GHz}$ to $+6 \mathrm{GHz}$ relative to the OI frequency. This LO pumps seven HEB mixers simultaneously. ${ }^{10}$ Since the first flight, many discoveries have been enabled with this LO, for example, the first detection of the OI line in the atmosphere of Mars. ${ }^{100}$ In Fig. 12, seven OI spectra of W49 N, a star-forming region about 36200 light years away from Earth, are shown. The spectra are measured simultaneously with the seven HEB mixers of the upGREAT array heterodyne spectrometer. ${ }^{11}$ Each spectrum corresponds to a different position in $\mathrm{W} 49 \mathrm{~N}$. Note the strong absorption along the line-of-sight toward W49 N, which is caused by the spiral arms of our galaxy. Emission from $\mathrm{W} 49 \mathrm{~N}$ is seen only at negative velocities just below $0 \mathrm{~km} / \mathrm{s}^{11,101}$

While the LO is very well suited for operation on SOFIA, there are a number of developments, which are required for balloon or spaceborne missions, such as those that are mentioned in Sec. I. With respect to the QCL itself, an improved frequency accuracy is required, in particular, because the atmospheric OI "reference" line is not available for a satellite instrument. A number of stabilization schemes have been discussed in Sec. III. From all

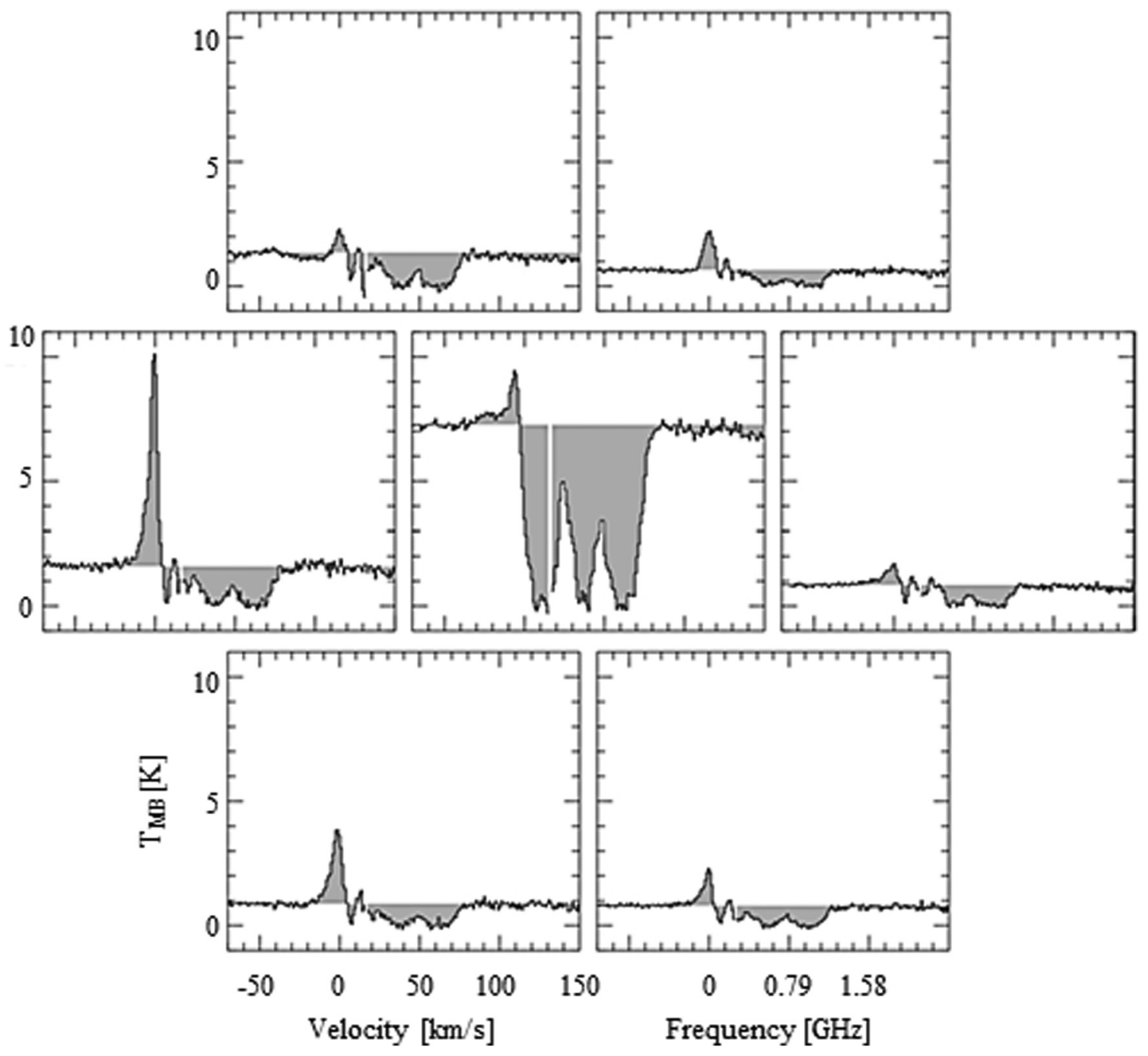

FIG. 12. Ol spectra at $4.7448 \mathrm{THz}$ observed with the upGREAT array heterodyne spectrometer on board of SOFIA. The spectra have been measured simultaneously with the seven pixels of the spectrometer. Each spectrum corresponds to a different position in W49 N. The relative positions of the spectra resemble the positions of the HEB mixers. The abscissa is given in terms of velocity and frequency relative to the rest frame of W49 N. The spectra are smoothed to a resolution of $1 \mathrm{~km} / \mathrm{s}(15.8 \mathrm{MHz})$. Adapted with permission from Risacher et al., J. Astron. Instrum. 7, 1840014 (2019). Copyright 2019 World Scientific Publishing Company. 
of these techniques, phase-locking to the harmonic of a multiplier source seems to be the most promising one, because it provides excellent frequency accuracy, requires little input power and little volume, and because this technology is space-proven. Another important issue is the frequency coverage or tuning range of a single QCL. If the application requires the observation of many molecular lines, a rather large frequency coverage, which easily exceeds $100 \mathrm{GHz}$, is required. For example, the fine structure line of OI is $120 \mathrm{GHz}$ above a rotational transition of $\mathrm{OH}$, and the two nearest rotational transitions of $\mathrm{CO}$ are $30 \mathrm{GHz}$ below and $85 \mathrm{GHz}$ above. Whether this frequency coverage can be achieved with a practical QCL LO remains to be demonstrated. Currently, one promising approach is LIFT, ${ }^{69}$ but it needs some technical modifications; in particular, the microscope objective should be replaced, for example, by fiber coupling [Fig. 7(c)]. ${ }^{68}$ An external cavity resonator is another possible way to go. Here, the THz VECSEL approach is particularly promising. ${ }^{81,82}$ However, broad frequency coverage and high spectral resolution need to be demonstrated by molecular spectroscopy. The output power requirement is governed by system aspects: Large HEB mixer arrays with up to 100 pixels are envisaged. With each pixel requiring about $1 \mu \mathrm{W}$ and with beam-splitter coupling of LO and signal radiation, i.e., about $99 \%$ of the LO radiation is lost at the beam splitter, an output power in the order of $10 \mathrm{~mW}$ is required. Similarly, with a Schottky diode mixer, which requires a few megawatts of LO power, at least $10 \mathrm{~mW}$ QCL power is required. The $\mathrm{LO}$ power requirement can be relaxed, if a diplexer is used instead of a beam splitter.

From a system point of view, mass, volume, and electrical input power of the LO system need to be taken into account. While the exact requirements depend on the particular mission, a few concerns apply virtually to all missions. The first is the power consumption. It is important to keep in mind that the wall plug efficiency of a QCL, which is often given in publications on a particular QCL, is not an appropriate figure-of-merit. Instead, the system wall plug efficiency is relevant. The typical input power of a QCL is a few watts. Cooling with liquid helium is not an option for any mission, because of complexity and the large amount of helium, which is necessary for cooling. Compact mechanical cryocoolers require in the order of $100 \mathrm{~W}$ electrical input power, much more than the QCL itself. Similar arguments hold for mass and volume considerations. Again, it is the cryocooler that drives the design. Therefore, when defining the LO system for a particular mission, the QCL and the cooling system have to be considered as a whole. A particular situation occurs for space missions. With passive cooling, it is possible to reach temperatures around $100 \mathrm{~K}$. QCLs, which operate at such temperatures with sufficient output power, would simplify the system significantly. However, there has been very little progress in this direction in the past decade. In addition, there are other constraints such as the mass and volume of the satellite radiators and orbit restrictions. Irrespective of that, QCLs have reached maturity, which makes space missions for astronomy and atmospheric science with a QCL LO feasible within the next few years.

\section{SUMMARY AND OUTLOOK}

In the past few years, many important techniques for highresolution absorption spectroscopy have been developed and demonstrated with THz QCLs, namely, direct absorption spectroscopy, detection schemes such as wavelength and frequency modulation, differential spectroscopy, photoacoustic spectroscopy, and heterodyne spectroscopy. In the latter case, the development of QCLs is very well advanced, as it is shown by the QCL LOs on board of SOFIA. Despite these successes, spectrometers with $\mathrm{THz}$ QCLs are not widespread, because a key enabling feature is missing. This is wide frequency tunability. A few $\mathrm{GHz}$ tuning range, which is available by changing the current and temperature of a QCL, is sufficient if a single or very few molecular transitions are of interest. However, in general, many lines have to be measured and the tuning range needs to be much larger. The development of LIFT is a very promising approach, because it combines a wide tuning range with easy implementation. A particularly noteworthy development is the THz QC-VECSEL, because it combines excellent beam profile with high output power. However, again, wide frequency tunability has to be demonstrated. Once the obstacle of frequency tunability is overcome, QCLs are likely to find many applications in exploring the $\mathrm{THz}$ region by molecular spectroscopy. This may also spur the development of more complex spectroscopy schemes, which are commonly used in other portions of the electromagnetic spectrum, for example, chirped laser spectroscopy or cavity ringdown spectroscopy. With respect to the latter technique, high-finesse $\mathrm{THz}$ resonators are required. ${ }^{102,103}$ Also, pulsed THz QCLs may find applications for cavity ringdown spectroscopy.

For real-world sensing applications, for example, in nondestructive testing or environmental sensing, photoacoustic detection as well as detection by optical feedback are promising approaches. However, in both cases, the sensitivity has to be improved and competition with established techniques, in particular, infrared spectroscopy, needs to be taken into account. Also, cryogenic cooling is a serious limitation, which prevents the use of $\mathrm{THz}$ QCLs in everyday applications. Unfortunately, during the past few years, progress in this direction has been small despite significant efforts. Whether this will change in the coming years remains to be seen. If cryogenic cooling is not an option, a possible alternative to $\mathrm{THz}$ QCLs is $\mathrm{THz}$ sources based on intracavity difference-frequency generation in mid-infrared QCLs. These have been shown to operate up to room temperature ${ }^{104}$ with high spectral purity and tenability, ${ }^{105}$ but the output power of around $10 \mu \mathrm{W}$ is rather low.

With respect to heterodyne spectroscopy, QCLs have demonstrated their potential on board of SOFIA. The availability of appropriate QCLs has enabled the observation of the important OI line. Similar systems are being developed for balloon-borne applications. With respect to spaceborne applications, operation temperatures of the QCLs are desirable, which are accessible by passive cooling. These temperatures are around $100 \mathrm{~K}$ depending on the electrical input power of the QCL.

Active frequency stabilization as well as increased frequency tuning are other important parameters, which need to be improved. In particular, for heterodyne spectrometers, which are part of largescale missions, it is important to keep in mind the system aspects, since these are the design drivers.

In view of past and possible future developments, one can expect that the true potential of $\mathrm{THz}$ QCLs for high-resolution molecular spectroscopy will be realized in the coming years. New 
and exciting discoveries in the field of molecular physics as well as in astronomy and atmospheric science will be enabled.

\section{REFERENCES}

1F. C. De Lucia, J. Mol. Spectrosc. 261, 1 (2010).

${ }^{2}$ S. Schlemmer, "High-resolution laboratory terahertz spectroscopy and applications to astrophysics," in Frontiers and Advances in Molecular Spectroscopy (Elsevier Science, Amsterdam, 2018), Vol. 471.

${ }^{3}$ H.-W. Hübers, IEEE J. Sel. Top. Quantum Electron. 14, 378 (2008).

${ }^{4}$ R. Eichholz, H. Richter, M. Wienold, L. Schrottke, R. Hey, H. T. Grahn, and H.-W. Hübers, Opt. Express 21, 32199 (2013).

${ }^{5}$ L. Consolino, S. Bartalini, H. E. Beere, D. A. Ritchie, M. S. Vitiello, and P. D. Natale, Sensors 13, 3331 (2013).

${ }^{6}$ S. Borri, P. Patimisco, A. Sampaolo, H. E. Beere, D. A. Ritchie, M. S. Vitiello, G. Scamarcio, and V. Spagnolo, Appl. Phys. Lett. 103, 021105 (2013).

${ }^{7}$ S. Bartalini, L. Consolino, P. Cancio, P. D. Natale, P. Bartolini, A. Taschin, M. De Pas, H. Beere, D. Ritchie, M. S. Vitiello, and R. Torre, Phys. Rev. X 4, 021006 (2014).

${ }^{8}$ M. Wienold, T. Alam, L. Schrottke, H. T. Grahn, and H.-W. Hübers, Opt. Express 26, 6692 (2018).

${ }^{9}$ H. Richter, M. Wienold, L. Schrottke, K. Biermann, H. T. Grahn, and H.-W. Hübers, IEEE Trans. Terahertz Sci. Technol. 5, 539 (2015).

${ }^{10}$ S. Heyminck, U. U. Graf, R. Güsten, J. Stuzki, H.-W. Hübers, and P. Hartogh, Astron. Astrophys. 542, L1 (2012).

${ }^{11}$ C. Risacher, R. Güsten, J. Stutzki, H.-W. Hübers, R. Aladro, A. Bell, C. Buchbender, D. Büchel, T. Csengeri, C. Duran, U. U. Graf, R. D. Higgins, C. E. Honingh, K. Jacobs, M. Justen, B. Klein, M. Mertens, Y. Okada, A. Parikka, P. Pütz, N. Reyes, H. Richter, O. Ricken, D. Riquelme, N. Rothbart, N. Schneider, R. Simon, M. Wienold, H. Wiesemeyer, M. Ziebart, P. Fusco, S. Rosner, and B. Wohler, J. Astron. Instrum. 7, 1840014 (2019).

${ }^{12}$ C. K. Walker, C. Kulesa, P. Goldsmith, C. Groppi, C. Helmich, D. Hollenbach, J. Kawamura, W. Langer, G. Melnick, D. Neufeld, J. Pineda, G. Stacey, A. Stark, A. Tielens, M. Wolfire, H. Yorke, and E. Young, "GUSTO: Gal/Xgal U/LDB spectroscopic-stratospheric terahertz observatory," in 231st American Astronomical Society (AAS) Meeting (AAS, 2018).

${ }^{13}$ S. P. Rea, B. N. Ellison, B. Swinyard, A. Valavanis, Y. Han, E. H. Linfield, A. G. Davies, C. Saunders, S. Parkes, D. Gerber, M. Henry, H. Wang, B. Alderman, O. Auriacombe, T. Rawlings, M. Crook, and T. Bradshaw, in Proceedings of the 26th International Symposium on Space Terahertz Technology, Cambridge, MA, 16-18 March 2015 (Harvard College Observatory, 2015), M1-3.

${ }^{14}$ D. Rigopoulou, M. Caldwell, B. Ellison, C. Pearson, E. Caux, A. Cooray, J. D. Gallego, M. Gerin, J. R. Goicoechea, P. Goldsmith, C. Kramer, D. C. Lis, S. Molinari, V. Ossenkopf-Okada, G. Savini, B. K. Tan, X. Tielens, S. Viti, M. Wiedner, and G. Yassin, Proc. SPIE 9904, 99042K (2016).

${ }^{15}$ D. Leisawitz, E. Amatucci, R. Carter, M. DiPirro, A. Flores, J. Staguhn, C. Wu, L. Allen, J. Arenberg, L. Armus, C. Battersby, J. Bauer, R. Bell, P. Beltran, D. Benford, E. Bergin, C. M. Bradford, D. Bradley, D. Burgarella, S. Carey, D. Chi, A. Cooray, J. Corsetti, E. De Beck, K. Denis, L. Dewell, M. East, S. Edgington, K. Ennico, L. Fantano, G. Feller, D. Folta, J. Fortney, J. Generie, M. Gerin, Z. Granger, G. Harpole, K. Harvey, F. Helmich, L. Hilliard, J. Howard, M. Jacoby, A. Jamil, T. Kataria, S. Knight, P. Knollenberg, P. Lightsey, S. Lipscy, E. Mamajek, G. Martins, M. Meixner, G. Melnick, S. Milam, T. Mooney, S. H. Moseley, D. Narayanan, S. Neff, T. Nguyen, A. Nordt, J. Olson, D. Padgett, M. Petach, S. Petro, J. Pohner, K. Pontoppidan, A. Pope, D. Ramspacher, T. Roellig, I. Sakon, C. Sandin, K. Sandstrom, D. Scott, K. Sheth, J. Steeves, K. Stevenson, L. Stokowski, E. Stoneking, K. Su, K. Tajdaran, S. Tompkins, J. Vieira, C. Webster, M. Wiedner, E. L. Wright, and J. Zmuidzinas, Proc. SPIE 10698, 1069815 (2018).

${ }^{16}$ M. C. Wiedner, I. Mehdi, A. Baryshev, V. Belitsky, V. Desmaris, A. DiGiorgio, J.-D. Gallego, M. Gerin, P. Goldsmith, F. Helmich, W. Jellema, A. Laurens, C. Risacher, A. Cooray, and M. Meixner, IEEE Trans. Terahertz Sci. Technol. 8, 558 (2018).
${ }^{17}$ G. A. Blake, K. B. Laughlin, R. C. Cohen, K. L. Busarow, D.-H. Gwo, C. A. Schmuttenmaer, D. W. Steiert, and R. J. Saykally, Rev. Sci. Instrum. 62, 1701 (1991).

${ }^{18}$ G. Cazzoli and C. Puzzarini, J. Phys. Chem. A 117, 13759 (2013).

${ }^{19}$ J. C. Pearson, B. J. Drouin, A. Maestrini, I. Mehdi, J. Ward, R. H. Lin, S. Yu, J. J. Gill, B. Thomas, C. Lee, G. Chattopadhyay, E. Schlecht, F. W. Maiwald, P. F. Goldsmith, and P. Siegel, Rev. Sci. Instrum. 82, 093105 (2011).

${ }^{20}$ S. Alighanbari, M. G. Hansen, V. I. Korobov, and S. Schiller, Nat. Phys. 14, 555 (2018).

${ }^{21}$ H.-W. Hübers, H. Richter, R. Eichholz, M. Wienold, K. Biermann, L. Schrottke, and H. T. Grahn, IEEE J. Sel. Top. Quantum Electron. 23, 1 (2017).

${ }^{22}$ B. S. Williams, Nat. Photonics 1, 517 (2007).

${ }^{23}$ G. Scalari, C. Walther, M. Fischer, R. Terazzi, H. Beere, D. Ritchie, and J. Faist, Laser Photonics Rev. 3, 45 (2009).

${ }^{\mathbf{2 4}}$ M. S. Vitiello, G. Scalari, B. Williams, and P. D. Natale, Opt. Express 23, 5167 (2015).

${ }^{25}$ J. Faist, Quantum Cascade Lasers (Oxford University Press, Oxford, 2013).

${ }^{26}$ L. H. Li, L. Chen, J. R. Freeman, M. Salih, P. Dean, A. G. Davies, and E. H. Linfield, Electron. Lett. 53, 799 (2017).

${ }^{27}$ M. Wienold, L. Schrottke, M. Giehler, R. Hey, and H. T. Grahn, J. Appl. Phys. 109, 073112 (2011)

${ }^{28}$ R. S. Dhar, S. G. Razavipour, E. Dupont, C. Xu, S. Laframboise, Z. Wasilewski, Q. Hu, and D. Ban, Sci. Rep. 4, 7183 (2014).

${ }^{29}$ D. O. Winge, E. Dupont, and A. Wacker, Phys. Rev. A 98, 023834 (2018).

${ }^{30}$ B. S. Williams, S. Kumar, Q. Hu, and J. L. Reno, Electron. Lett. 42, 89 (2006).

${ }^{31}$ S. Fathololoumi, E. Dupont, C. W. I. Chan, Z. R. Wasilewski, S. R. Laframboise, D. Ban, A. Mátyás, C. Jirauschek, Q. Hu, and H. C. Liu, Opt. Express 20, 3866 (2012).

${ }^{32}$ S. Barbieri, J. Alton, H. E. Beere, J. Fowler, E. H. Linfield, and D. A. Ritchie, Appl. Phys. Lett. 85, 1674 (2004).

${ }^{33}$ M. Wienold, L. Schrottke, M. Giehler, R. Hey, W. Anders, and H. T. Grahn, Electron. Lett. 45, 1030 (2009).

${ }^{34}$ M. I. Amanti, G. Scalari, R. Terazzi, M. Fischer, M. Beck, J. Faist, A. Rudra, P. Gallo, and E. Kapon, New J. Phys. 11, 125022 (2009).

${ }^{35}$ L. Schrottke, M. Wienold, R. Sharma, X. Lü, K. Biermann, R. Hey, A. Tahraoui, H. Richter, H.-W. Hübers, and H. T. Grahn, Semicond. Sci. Technol. 28, 035011 (2013).

${ }^{36}$ L. Schrottke, X. Lü, G. Rozas, K. Biermann, and H. T. Grahn, Appl. Phys. Lett. 108, 102102 (2016).

${ }^{37}$ K. Ohtani, D. Turčinková, C. Bonzon, I.-C. Benea-Chelmus, M. Beck, J. Faist, M. Justen, U. U. Graf, M. Mertens, and J. Stutzki, New J. Phys. 18, 123004 (2014).

${ }^{38} \mathrm{M}$. Wienold, B. Röben, L. Schrottke, R. Sharma, A. Tahraoui, K. Biermann, and H. T. Grahn, Opt. Express 22, 3334 (2014).

${ }^{39}$ A. J. L. Adam, I. Kašalynas, J. N. Hovenier, T. O. Klaassen, J. R. Gao, E. E. Orlova, B. S. Williams, S. Kumar, Q. Hu, and J. L. Reno, Appl. Phys. Lett. 88, 151105 (2006).

${ }^{40}$ M. I. Amanti, M. Fischer, G. Scalari, M. Beck, and J. Faist, Nat. Photonics 3, 586 (2009).

${ }^{41}$ L. Mahler and A. Tredicucci, Laser Photon. Rev. 5, 647 (2011).

${ }^{42}$ C. Sirtori, S. Barbieri, and R. Colombelli, Nat. Photonics 7, 691 (2013).

${ }^{43}$ D. Turčinková, M. I. Amanti, F. Castellano, M. Beck, and J. Faist, Appl. Phys. Lett. 102, 181113 (2013).

${ }^{44}$ L. Bosco, C. Bonzon, K. Ohtani, M. Justen, M. Beck, and J. Faist, Appl. Phys. Lett. 109, 201103 (2016).

${ }^{45} \mathrm{X}$. Wang, C. Shen, T. Jiang, Z. Zhan, Q. Deng, W. Li, W. Wu, N. Yang, W. Chu, and S. Duan, AIP Adv. 6, 075210 (2016).

${ }^{46}$ A. L. Betz, R. T. Boreiko, B. S. Williams, S. Kumar, Q. Hu, and J. L. Reno, Opt. Lett. 30, 1837 (2005).

${ }^{47}$ A. A. Danylov, T. M. Goyette, J. Waldman, M. J. Coulombe, A. J. Gatesman, R. H. Giles, W. D. Goodhue, X. Qian, and W. E. Nixon, Opt. Express 17, 7525 (2009). ${ }^{48}$ D. Rabanus, U. U. Graf, M. Philipp, O. Ricken, J. Stutzki, B. Vowinkel, M. C. Wiedner, C. Walther, M. Fischer, and J. Faist, Opt. Express 17, 1159 (2009). 
${ }^{49}$ D. J. Hayton, A. Khudchencko, D. G. Pavelyev, J. N. Hovenier, A. Baryshev, J. R. Gao, T.-Y. Kao, Q. Hu, J. L. Reno, and V. Vaks, Appl. Phys. Lett. 103, 051115 (2013).

${ }^{50}$ B. T. Bulcha, J. L. Hesler, V. Drakinskiy, J. Stake, A. Valavanis, P. Dean, L. H. Li, and N. S. Barker, IEEE Trans. Terahertz Sci. Technol. 6, 737 (2016).

${ }^{51}$ S. Barbieri, P. Gellie, G. Santarelli, L. Ding, W. Maineult, C. Sirtori, R. Colombelli, H. Beere, and D. Ritchie, Nat. Photonics 4, 636 (2010).

52 J. Freeman, L. Ponnampalam, H. Shams, R. A. Mohandas, C. C. Renaud, P. Dean, L. Li, A. G. Davies, A. J. Seeds, and E. H. Linfield, Optica 4, 1059 (2017).

${ }^{53}$ G. Moruzzi, P. Riminucci, F. Strumia, B. Carli, M. Carlotti, R. M. Lees, I. Mukhopadhyay, J. W. C. Johns, B. P. Winnewisser, and M. Winnewisser, J. Mol. Spectrosc. 144, 139 (1990).

${ }^{54}$ T. Varberg, L. Zing, and K. Evenson, Astrophys. J. 385, 763 (1992)..

${ }^{55}$ H. Richter, S. G. Pavlov, A. D. Semenov, L. Mahler, A. Tredicucci, H. E. Beere, D. A. Ritchie, and H.-W. Hübers, Appl. Phys. Lett. 96, 071112 (2010).

${ }^{\mathbf{5 6}}$ Y. Ren, J. N. Hovenier, M. Cui, D. J. Hayton, J. R. Gao, T. M. Klapwijk, S. C. Shi, T.-Y. Kao, Q. Hu, and J. L. Reno, Appl. Phys. Lett. 100, 041111 (2012).

${ }^{57}$ J. Xu, J. M. Hensley, D. B. Fenner, R. P. Green, L. Mahler, A. Tredicucci, M. G. Allen, F. Beltram, H. E. Beere, and D. A. Ritchie, Appl. Phys. Lett. 91, 121104 (2007).

${ }^{58}$ A. W. M. Lee, B. S. Williams, S. Kumar, Q. Hu, and J. L. Reno, Opt. Lett. 35, $910(2010$

${ }^{59}$ C. A. Curwen, J. L. Reno, and B. S. Williams, Appl. Phys. Lett. 113, 011104 (2018).

${ }^{60}$ Q. Qin, B. S. Williams, S. Kumar, J. L. Reno, and Q. Hu, Nat. Photonics 3, 732 (2009).

${ }^{61}$ N. Han, A. D. Geofroy, D. P. Burghoff, C. W. I. Chan, A. W. M. Lee, J. L. Reno, and Q. Hu, Opt. Lett. 39, 3480 (2014).

${ }^{62}$ K. Ohanti, M. Beck, and J. Faist, Appl. Phys. Lett. 104, 011107 (2014).

${ }^{63}$ I. Kundu, P. Dean, A. Valavanis, L. Chen, L. Li, J. E. Cunningham, E. H. Linfield, and A. G. Davies, Opt. Express 22, 16595 (2014).

${ }^{64}$ D. Turčinková, M. I. Amanti, G. Scalari, M. Beck, and J. Faist, Appl. Phys. Lett. 106, 131107 (2015).

${ }^{65}$ I. Kundu, P. Dean, A. Valavanis, J. R. Freeman, M. C. Rosamond, L. Li, Y. Han, E. H. Linfield, and A. G. Davies, ACS Photonics 5, 2912 (2018).

${ }^{66}$ T. Hagelschuer, M. Wienold, H. Richter, L. Schrottke, H. T. Grahn, and H.-W. Hübers, Opt. Express 25, 30203 (2017).

${ }^{67}$ M. Hempel, B. Röben, L. Schrottke, H.-W. Hübers, and H. T. Grahn, Appl. Phys. Lett. 108, 191106 (2016)

${ }^{68}$ M. Hempel, B. Röben, M. Niehle, L. Schrottke, A. Trampert, and H. T. Grahn, AIP Adv. 7, 059903 (2017).

${ }^{69}$ T. Alam, M. Wienold, X. Lü, K. Biermann, L. Schrottky, H. T. Grahn, and H.-W. Hübers, Opt. Express 27, 5420 (2019).

${ }^{70}$ H.-W. Hübers, S. G. Pavlov, A. D. Semenov, R. Köhler, L. Mahler, A. Tredicucci, H. E. Beere, D. A. Ritchie, and E. H. Linfield, Opt. Express 13, 5890 (2005).

${ }^{71}$ E. Bründermann, M. Havenith, G. Scalari, M. Giovannini, J. Faist, J. Kunsch, L. Mechold, and M. Abraham, Opt. Express 14, 1829 (2006).

${ }^{72}$ H. Richter, A. D. Semenov, S. G. Pavlov, L. Mahler, A. Tredicucci, H. E. Beere, D. A. Ritchie, K. S. Il'in, M. Siegel, and H.-W. Hübers, Appl. Phys. Lett. 93, 141105 (2008).

${ }^{73}$ A. W. M. Lee, Q. Qin, S. Kumar, B. S. Williams, Q. Hu, and J. L. Reno, Opt. Lett. 32, 2840 (2007).

${ }^{74}$ M. I. Amanti, M. Fisher, C. Walther, G. Scalari, and J. Faist, Electron. Lett. 43, 573 (2007).

${ }^{75}$ M. Justen, K. Otani, D. Turčinková, F. Castellano, M. Beck, U. U. Graf, D. Büchel, M. Schultz, and J. Faist, IEEE J. Sel. Top. Quantum Electron. 7, 609 (2017).

${ }^{76}$ S. Kumar, B. S. Williams, Q. Qin, A. W. Lee, Q. Hu, and J. L. Reno, Opt. Express 15, 113 (2007).

${ }^{\text {77}}$ T.-Y. Kao, Q. Hu, and J. L. Reno, Opt. Lett. 37, 2070 (2012).
${ }^{78}$ Y. Chassagneux, R. Colombelli, W. Maineult, S. Barbieri, H. E. Beere, D. A. Ritchie, S. P. Khanna, E. H. Linfield, and A. G. Davies, Nature 457, 174 (2009). ${ }^{79} \mathrm{G}$. Xu, R. Colombelli, S. P. Khanna, A. Belarouci, X. Letartre, L. Li, E. H. Linfield, A. G. Davies, H. E. Beere, and D. A. Ritchie, Nat. Commun. 3 (2012), 952 (2012).

${ }^{80}$ T.-Y. Kao, Q. Hu, and J. L. Reno, Appl. Phys. Lett. 96, 101106 (2010).

${ }^{81}$ L. Xu, C. A. Curwen, P. W. C. Hon, Q.-S. Chen, T. Itoh, and B. S. Williams, Appl. Phys. Lett. 107, 221105 (2015).

${ }^{82}$ L. Xu, D. Chen, T. Itoh, J. L. Reno, and B. S. Williams, Opt. Express 24, 24117 (2016).

${ }^{83}$ H.-W. Hübers, S. G. Pavlov, H. Richter, A. D. Semenov, L. Mahler, A. Tredicucci, H. E. Beere, and D. A. Ritchie, Appl. Phys. Lett. 89, 061115 (2006).

${ }^{84}$ L.-H. Xu, J. Fisher, R. M. Lees, H. Y. Shi, J. T. Hougen, J. C. Pearson, B. J. Drouin, G. A. Blake, and R. Braakmann, J. Mol. Spectrosc. 251, 305 (2008).

${ }^{85}$ R. Eichholz, H. Richter, S. G. Pavlov, M. Wienold, L. Schrottke, R. Hey, H. T. Grahn, and H.-W. Hübers, Appl. Phys. Lett. 99, 141112 (2011).

${ }^{86}$ B. Röben, X. Lü, M. Hempel, K. Biermann, L. Schrottke, and H. T. Grahn, Opt. Express 25, 16282 (2017).

${ }^{87}$ D. Burghoff, T.-Y. Kao, N. Han, C. W. I. Chan, X. Cai, Y. Yang, D. J. Hayton, J.-R. Gao, J. L. Reno, and Q. Hu, Nat. Photonics 8, 462 (2014).

${ }^{88}$ M. Rösch, G. Scalari, G. Villares, L. Bosco, M. Beck, and J. Faist, Appl. Phys. Lett. 108, 171104 (2016).

${ }^{89}$ Y. Yang, D. Burghoff, D. J. Hayton, J.-R. Gao, J. L. Reno, and Q. Hu, Optica 3, 499 (2016).

${ }^{90}$ M. Rösch, G. Scalari, M. Beck, and J. Faist, Nat. Photonics 9, 42 (2015).

${ }^{91}$ L. A. Sterczewski, J. Westberg, Y. Yang, D. Burghoff, J. Reno, Q. Hu, and G. Wysocki, "Terahertz hyperspectral imaging with dual chip-scale combs," e-print arXiv:1812.03505 (2019).

${ }^{92}$ A. Sampaolo, P. Patimisco, M. Giglio, M. S. Vitiello, H. E. Beere, D. A. Ritchie, G. Scamarcio, F. K. Tittel, and V. Spagnolo, Sensors 16, 439 (2016).

${ }^{93}$ T. Hagelschuer, N. Rothbart, H. Richter, M. Wienold, L. Schrottke, H. T. Grahn, and H.-W. Hübers, Opt. Express 24, 13839 (2016).

${ }^{94}$ L. Consolino, A. Campa, M. Ravaro, D. Mazzotti, M. S. Vitiello, S. Bartalini, and P. De Natale, Appl. Phys. Lett. 106, 021108 (2015).

${ }^{95}$ M. S. Vitiello, L. Consolino, S. Bartalini, A. Taschin, A. Tredicucci, M. Inguscio, and P. D. Natale, Nat. Photonics 6, 525 (2012).

${ }^{96}$ J. R. Gao, J. N. Hovenier, Z. Q. Yang, J. J. A. Baselmans, A. Baryshev, M. Hajenius, T. M. Klapwijk, A. J. L. Adam, T. O. Klaassen, B. S. Williams, S. Kumar, Q. Hu, and J. L. Reno, Appl. Phys. Lett. 86, 244104 (2005).

${ }^{97}$ H. Richter, M. Greiner-Bär, S. G. Pavlov, A. D. Semenov, M. Wienold, L. Schrottke, M. Giehler, R. Hey, H. T. Grahn, and H.-W. Hübers, Opt. Express 18, 10177 (2010).

${ }^{98}$ R. Y. Ren, J. N. Hovenier, R. Higgins, J. R. Gao, T. M. Klapwijk, S. C. Shi, B. Klein, T.-Y. Kao, Q. Hu, and J. L. Reno, Appl. Phys. Lett. 98, 231109 (2011).

${ }^{99} \mathrm{M}$. Wienold, A. Tahraoui, L. Schrottke, R. Sharma, X. Lü, K. Biermann, R. Hey, and H. T. Grahn, Opt. Express 20, 11207 (2012).

${ }^{100}$ L. Rezac, P. Hartogh, R. Güsten, H. Wiesemeyer, H.-W. Hübers, C. Jarchow, H. Richter, B. Klein, and N. Honingh, Astron. Astrophys. 580, L10 (2015).

${ }^{{ }^{101}} \mathrm{H}$. Wiesemeyer, R. Güsten, S. Heyminck, H. W. Hübers, K. M. Menten, D. A. Neufeld, H. Richter, R. Simon, J. Stutzki, B. Winkel, and F. Wyrowski, Astron. Astrophys. 585, A76 (2016).

${ }^{102}$ R. Braakman and G. A. Blake, J. Appl. Phys. 109, 063102 (2011).

${ }^{103}$ L. Consolino, A. Campa, D. Mazzotti, M. S. Vitiello, P. De Natale, and S. Bartalini, Photonics 6, 1 (2019).

${ }^{104}$ Q. Lu, D. Wu, S. Sengupta, S. Slivken, and M. Razeghi, Sci. Rep. 6, 23595 (2016).

${ }^{105}$ L. Consolino, S. Jung, A. Campa, M. De Regis, S. Pal, J. H. Kim, K. Fujita, A. Ito, M. Hitaka, S. Bartalini, P. De Natale, M. A. Belkin, and M. S. Vitiello, Sci. Adv. 3, el603317 (2017). 